\title{
Priming Strategies for Benefiting Plant Performance under Toxic Trace Metal Exposure
}

\begin{abstract}
Alina Wiszniewska
check for updates

Department of Botany, Physiology and Plant Protection, Faculty of Biotechnology and Horticulture, University of Agriculture in Kraków, Al. 29 Listopada 54, 31-425 Kraków, Poland; a.wiszniewska@urk.edu.pl

Abstract: Combating environmental stress related to the presence of toxic elements is one of the most important challenges in plant production. The majority of plant species suffer from developmental abnormalities caused by an exposure to toxic concentrations of metals and metalloids, mainly $\mathrm{Al}$, As, $\mathrm{Cd}, \mathrm{Cu}, \mathrm{Hg}, \mathrm{Ni}, \mathrm{Pb}$, and $\mathrm{Zn}$. However, defense mechanisms are activated with diverse intensity and efficiency. Enhancement of defense potential can be achieved though exogenously applied treatments, resulting in a higher capability of surviving and developing under stress and become, at least temporarily, tolerant to stress factors. In this review, I present several already recognized as well as novel methods of the priming process called priming, resulting in the so-called "primed state" of the plant organism. Primed plants have a higher capability of surviving and developing under stress, and become, at least temporarily, tolerant to stress factors. In this review, several already recognized as well as novel methods of priming plants towards tolerance to metallic stress are discussed, with attention paid to similarities in priming mechanisms activated by the most versatile priming agents. This knowledge could contribute to the development of priming mixtures to counteract negative effects of multi-metallic and multi-abiotic stresses. Presentation of mechanisms is complemented with information on the genes regulated by priming towards metallic stress tolerance. Novel compounds and techniques that can be exploited in priming experiments are also summarized.
\end{abstract}

Citation: Wiszniewska, A. Priming Strategies for Benefiting Plant Performance under Toxic Trace Metal Exposure. Plants 2021, 10, 623. https://doi.org/10.3390/ plants10040623

Academic Editor: Magda Pál

Received: 9 March 2021

Accepted: 22 March 2021

Published: 25 March 2021

Publisher's Note: MDPI stays neutral with regard to jurisdictional claims in published maps and institutional affiliations.

Copyright: (C) 2021 by the author. Licensee MDPI, Basel, Switzerland. This article is an open access article distributed under the terms and conditions of the Creative Commons Attribution (CC BY) license (https:// creativecommons.org/licenses/by/ $4.0 /)$.
Keywords: chemical priming; induced acclimation; priming cocktail; soil pollution; toxic metals and metalloids

\section{Introduction}

Excessive amounts of toxic trace metal elements (heavy metals), accumulating in the soil, air, and water due to anthropogenic activities negatively affect environmental balance worldwide. In polluted land areas, toxic metals are absorbed by plants and stored in their tissues, entering the biocenotic food chains of both natural and agricultural characters. This contributes to diminished vegetable food quality because of heavy metal contamination and becomes a serious threat to human health [1]. Exposure to toxic metals itself causes growth disturbances and developmental abnormalities in the majority of plant species, namely, inhibited biomass accretion, overproduction of radicals due to impaired oxidative status, reduced photosynthetic performance, as well as changes in macromolecule synthesis and activity [2,3]. As plants are not capable of escaping stress due to their sessile form of living, they need to activate numerous defense mechanisms to counteract toxic implications of metallic stress. Some species, obligatory or facultative metallophytes, have developed particular adaptations, allowing them to grow and reproduce in heavy metal-contaminated environments [4-6]. Among them are hyperaccumulators capable of accumulating large amounts of toxic metals from the soil $[7,8]$. The whole organism responds to this environmental stress by neutralizing oxidative impairments as a result of enhanced antioxidant activity and also by phytohormonal signaling $[9,10]$. Metal transport to photosynthetic organs may be blocked, and then toxic ions remain stabilized in the belowground parts of the plant. At a cellular level, sequestration of toxic ions in the vacuoles occurs, accompanied by synthesis of metal-chelating compounds and ion transporters $[3,11]$. The ability of 
plants to survive and maintain proper growth under heavy metal exposure is conditioned by the collective action of these critical mechanisms.

Defense response in the presence of metallic stress can be activated with distinct rapidity and efficiency, thus diversifying the tolerance level. Such stress defense potential can be induced though exogenously applied treatments in a process called priming, resulting in the so-called "primed state" of the plant organism. Primed plants are considered acclimated to stressful environmental conditions and are able to survive and perform better than non-primed ones. Priming has been extensively studied and applied to enhance plant tolerance to pathogen attack and to numerous abiotic stresses, predominantly drought and water deficit [12-15]. Encouraging results have provoked the opinion that plant priming towards environmental stress tolerance is among the most promising research fields in the near future [16].

For this reason, the aim of this compact review is to present several already recognized as well as novel methods of priming plants towards an induced acclimation to metallic stress and to critically evaluate their priming mechanisms and applicability at the both laboratory scale and in plant production practice. It was also intended to discuss future directions in this area, mainly in relation to novel compounds and techniques that can be exploited in priming experiments to enhance plant tolerance to toxic metals. Complex information on the genetic background of priming mechanisms was also gathered to facilitate the designing of future studies involving manipulations on gene expression.

\section{What Does Priming Really Mean? The Aspects of Priming, Acclimation, and Hormesis}

In studies on plant physiology, the term priming usually means short-lasting preconditioning or pretreatment with specific compounds, biological agents or physical factors in order to achieve enhanced survivability and efficient action of defense mechanisms under subsequently encountered stress $[17,18]$. In contrast, defense responses developed during long periods of stress duration (chronic stress, often of a gradually increasing intensity), to which plant metabolism adjusts, are attributed to the acclimation phenomenon [15,19]. Acclimated plants maintain a higher content of stress-protective compounds and therefore are well equipped for subsequent stress episodes. In this respect, priming is considered advantageous over constitutive acclimation due to its resource-saving character [15]. Priming stimulus does not modify the DNA structure permanently, and therefore its effects are reversible. Stress memory is attributed to epigenetic changes in chromatin, accumulation of inactive transcription factors, and inactive signaling compounds [20].

A primed state is defined as the physiological status in which plants are capable of quicker and more effective activating stress-protective responses $[17,21]$. It is a kind of "state of readiness" to another stress event, of which the duration depends on numerous factors, including the type of stress stimulus, priming agent, and physiological condition of the plant organism. Priming factors can either trigger stress itself or be a compound or stimulus that acts as a stress predictor. In this respect, priming towards metallic stress tolerance is usually achieved via trans-priming. This refers to the induction of defense response compounds other than toxic metals [20]. Most of these substances are used to stimulate plant antioxidant capacity and to counteract oxidative damage caused by heavy metal exposure [22]. Cis-priming takes place when both the priming stimulus and response triggering stimulus are of the same character, for instance, a short-term water deficit enhances a plant's ability to survive subsequent drought [23]. In the case of toxic metal tolerance, such an approach may have confusing implications since plants subjected to transient metallic stress, particularly of low or mild intensity, exhibit a socalled hormetic effect [24-26]. This term refers to growth stimulation and overall better physiological performance after the application of low non-lethal doses of a toxic agent [27]. It is postulated that hormesis is an adaptive reaction to stress that allows compensating for a loss of internal balance in the poisoned organism [28]. Exploitation of intoxicated hormetic effect towards heavy metal tolerance is problematic due to environmental anxieties, and the same applies to cis-priming with trace metal ions. However, practical benefits in 
the form of enhanced survival during ionic stress can be obtained by priming plants with the alkali metals $\mathrm{Na}^{+}$and $\mathrm{K}^{+}[29,30]$. In turn, transition metal ions, particularly those regarded as essential elements, can be successfully applied as priming agents to provoke defense responses to other stresses, including drought [31-33], salinity [30,34-36], oxidative stress [37,38], as well as biotic stress [39] (Supplementary Table S1). This approach is supported by the evidence of metal involvement in cross tolerance to unfavorable conditions, even at an ecosystem level [40,41].

\section{Priming at Critical Growth Stages}

Priming can be applied at various developmental stages of the plant life cycle and to various plant parts. The most frequently used is seed priming that serves as a germination synchronizer and has numerous advantages from an agricultural viewpoint. The germination rate of seeds soaked in water prior to sowing is usually enhanced, and seedlings grow vigorously, uniformly developing their root system [42]. These features have a significant impact on further plant growth and performance, particularly on the crop yield. Seed priming by hydration and drying was developed to increase the quality of seeds and thus to enhance their agricultural value. The simplicity of seed treatment allows wide exploitation of the technique. During soaking, seeds bind water and absorb protective and biologically active compounds. As a result, the procedure activates various enzymes, metabolic pathways, and synthesis processes that are regarded as "pre-germinative metabolism" [43,44]. Promoting effects are then manifested in developing seedlings and sustain their higher vigor and survivability under unfavorable environmental conditions. As advanced priming techniques emerged, such as nano-priming and magneto-priming, obtaining plants that are tolerant to both biotic and abiotic stresses, became even more efficient [45,46]. Moreover, storage of seeds is more convenient as there is no need for dehydration after treatment [47].

Less often, priming is applied to seedlings or their parts, as well as young plants in active growth phases. This approach allows focusing on amelioration/evaluation of defense responses in specific plant regions or organs, for instance, the root system [48,49]. In the case of metallic stress tolerance/defense, pretreatment with plant growth regulators (PGR), called hormopriming, is conducted on seedlings and young and mature plants rather than on seeds [50].

Priming can be applied to organs or their fragments excised from donor plants using in vitro culture. Such an approach is rather labor-consuming and requires laboratory equipment necessary to establish proliferating cultures. An inevitable advantage is the possibility of wide-scale screening of priming compounds in relation to their form or concentration, with limited factors that may interfere with the results [48,51-53]. Since tissue culture conditions can be regarded as a stressful environment, priming helps to induce and sustain proliferation activity, leading to efficient plant regeneration. Priming can be performed in the form of specific manipulations during culture, application of chemical compounds to culture medium or explant pretreatment prior to culture initiation, or as culture biotization with growth-promoting microorganisms [54]. The main purposes of in vitro priming is stimulation of morphogenetic responses of explants, as well as synthesis and release of biologically active compounds [55-57]. These goals can be obtained using priming agents of physical, chemical, and biotic natures, as summarized in Table 1. Studies considering stress response show that various priming agents are capable of reprogramming plant metabolism, by inducing oxidative bursts or regulating key genes of secondary metabolites biosynthesis. This facilitates obtaining plants resistant to pathogens via regeneration from selected cell lines [58-60]. Considering metallic stress, in vitro culture systems focus on developing plant tolerance to chronic exposure to trace metals, contributing to the acquisition of an acclimation status. As a result of prolonged selection in the presence of stress agents, such as salinity or toxic elements, functioning of plant cells and regenerated plantlets is modified in comparison with non-exposed ones [19,61,62]. A hypothetical "priming agent" that induces acclimation to heavy metals in such culture systems has not been identified. A recent in vitro study revealed that application of a 
nitric oxide (NO) donor to tobacco plants cultured in vitro in the presence of $\mathrm{Cd}$ or $\mathrm{Cu}$ ameliorated growth by elevating rubisco and rubisco activase activity in comparison with plants treated with toxic metals without NO pretreatment [63]. Notwithstanding, reports evaluating typical priming effects in in vitro culture are still scarce due to the novelty of this concept.

Table 1. Examples of in vitro culture priming towards plant improvement and stress tolerance.

\begin{tabular}{|c|c|c|c|c|}
\hline Priming Agent & Plant Species & Culture Type & Priming Benefits & Reference \\
\hline $\begin{array}{l}6.3 \mu \mathrm{M} \text { chlorocholine } \\
\text { chloride }\end{array}$ & Eclipta alba & Shoot culture & $\begin{array}{c}\uparrow \text { root number and length } \\
\uparrow \text { biomass of regenerated plantlets } \\
\uparrow \text { chlorophyll content } \\
\text { 个survival after acclimatization to ex } \\
\text { vitro conditions }\end{array}$ & [56] \\
\hline $\begin{array}{c}1 \mathrm{mg} \cdot \mathrm{mL}^{-1} \text { ulvan (sulphated } \\
\text { polysaccharide from green } \\
\text { macroalga Ulva fasciata) }\end{array}$ & $\begin{array}{l}\text { Triticum aestivum } \\
\text { Oryza sativa } \\
\text { Hordeum vulgare }\end{array}$ & Suspension culture & $\begin{array}{l}\text { prevention from oxidative burst } \\
\text { caused by chitosan } \\
\downarrow \text { symptoms of infection with } \\
\text { pathogen Blumeria graminis }\end{array}$ & [58] \\
\hline Imprimatins & Arabidopsis thatiana & Suspension culture & $\begin{array}{c}\text { suppressed growth of bacterial } \\
\text { pathogens } \\
\uparrow \text { level of endogenous SA } \\
\uparrow \text { expression of PR genes } \\
\downarrow \text { reduced SA inactivation by } \\
\text { glucosylation }\end{array}$ & [48] \\
\hline
\end{tabular}

Pathogen-derived compounds (1 mM isonitroacetophenon, $300 \mu \mathrm{M}$ acibenzolar-S-methyl, 200 $\mathrm{nM}$ flagellin, $100 \mu \mathrm{g} \cdot \mathrm{mL}^{-1}$

chitosan, $100 \mu \mathrm{g} \cdot \mathrm{mL}^{-1}$

lipopolysaccharide)

Ultraviolet C (UVC) Coleus forskohlii Callus culture

callus organogenesis

$\uparrow$ resistance to Macrophomina root rot disease (M. phaseolina pathogen)

$\uparrow$ forskolin synthesis via upregulation of forskolin biosynthesis genes

\begin{tabular}{|c|c|c|c|c|}
\hline $\begin{array}{c}0.05 \mathrm{mM} \text { SNP (nitric oxide } \\
\text { donor) }\end{array}$ & Nicotiana tabacum & Shoot culture & $\begin{array}{l}\uparrow \text { tolerance to } \mathrm{Cd} \text { and } \mathrm{Cu} \text {, manifested } \\
\text { by higher biomass, chlorophyll } \\
\text { content and rubisco activity }\end{array}$ & [63] \\
\hline $200 \mathrm{nM}$ melatonin & Nicotiana tabacum & Suspension culture & $\uparrow$ cell survival in the presence of $\mathrm{Pb}$ & {$[51]$} \\
\hline $\begin{array}{l}\text { Volatile compounds released } \\
\text { by Microbacterium strain EC8 }\end{array}$ & $\begin{array}{l}\text { Arabidopsis thatiana } \\
\text { Lactuca sativa } \\
\text { Solanum } \\
\text { lycopersicum }\end{array}$ & $\begin{array}{l}\text { In vitro-grown } \\
\text { seedlings }\end{array}$ & $\begin{array}{l}\uparrow \text { growth promotion-shoot and root } \\
\text { biomass, lateral root density }\end{array}$ & [52] \\
\hline $\begin{array}{c}\text { Cold plasma } \\
5-80 \mathrm{mg} \cdot \mathrm{L}^{-1} \mathrm{SiO}_{2} \\
\text { nanoparticles }(\mathrm{nSi})\end{array}$ & Astragalus fridae & $\begin{array}{l}\text { Seeds germinating } \\
\text { in vitro }\end{array}$ & $\begin{array}{c}\uparrow \text { root system development } \\
\uparrow \text { shoot biomass } \\
\uparrow \text { chlorophyll and carotenoid contents } \\
\uparrow \text { root NR activity, shoot CAT activity } \\
\text { modified anatomy and tissue } \\
\text { differentiation patterns } \\
\text { interactive effect of cold plasma } \\
\text { and nSi }\end{array}$ & [53] \\
\hline
\end{tabular}

$\downarrow$ decrease/reduction; $\uparrow$ increase/enhancement. Abbreviations: CAT—catalase, NR—nitrate reductase, PRs—pathogen related genes, SA—salicylic acid, SNP—sodium nitroprusside 


\section{Major Players in Chemical Priming towards Toxic Metal(Loid) Tolerance}

Chemical priming consists of pretreatment with chemical compounds of natural or synthetic origin. Substances may be of a versatile chemical nature, from simple, purified compounds to multi-compound mixtures and extracts. The vast reports on chemical priming towards tolerance to metallic stress employ compounds involved in signaling and modulation of antioxidant response. It is a fruitful priming approach towards trace metal tolerance since enhanced activity of antioxidant machinery is crucial for compensation of oxidative imbalances caused by exposure to toxic metal ions.

Small signaling compounds (SSC), such as hydrogen peroxide $\left(\mathrm{H}_{2} \mathrm{O}_{2}\right)$, gasotransmitters-nitric oxide (NO), and hydrogen sulfide $\left(\mathrm{H}_{2} \mathrm{~S}\right)$, and phytohormone salicylic acid (SA) are the most frequently applied to induce priming effects in plants, regardless of the stress considered. These compounds trigger stress signals and activate defense mechanisms in a compound-specific manner; however, their common feature is the capability of inducing radical scavenging and antioxidant capacity. Moreover, all these substances interfere with each other, down- or up-regulating their quantity and metabolic pathways. Exogenously applied, the compounds influence the pool of their endogenous forms, and their orchestrated action contributes to the development of stress defense response.

Existing relationships and cross-talks between SSC have been excellently reviewed in several important papers [64-69], and interested readers can find comprehensive information. However, a detailed survey on the interactions of the four compounds taking place during heavy metal exposure is still missing. In this compact review, the results of applications of the respective compounds are presented in Table 2, with studies focusing on the interplays between at least two of them particularly highlighted. Small signaling compounds are the most versatile priming agents used for metallic stress tolerance induction, and the basics of their priming features are summarized below. Methodological details concerning pretreatments with small regulatory compounds in heavy metal-focused studies are summarized in Table 2.

Hydrogen peroxide. The most frequently reported is the priming effect of hydrogen peroxide $\left(\mathrm{H}_{2} \mathrm{O}_{2}\right)$, which in fact has prooxidant properties. Low doses of $\mathrm{H}_{2} \mathrm{O}_{2}$ induce a primed state in plants by exposing the organism to oxidative challenge of low intensity. During pretreatment, reactive oxygen species (ROS) are generated, leading to activation of ROS-dependent signaling networks. Subsequently, accumulation of defense proteins, including transcription factors and proteins actively involved in ROS scavenging, takes place, preparing the plant for forthcoming stress events [70]. The potential of $\mathrm{H}_{2} \mathrm{O}_{2}$ to counteract metal toxicity was comprehensively reviewed by [71], who discussed its involvement in signal transduction by oxidizing regulatory proteins, phytohormones, and their precursors, transcripts, as well as other signaling compounds. Priming effects of hydrogen peroxide are visible at low doses, ranging from 100 to $600 \mu \mathrm{M}$ (Table 2). At the level of plant growth and physiological status, the main benefits of $\mathrm{H}_{2} \mathrm{O}_{2}$ priming prior to metal treatment include decreased ROS production and oxidative damage of cell components. As a consequence, growth reduction is minimized [72,73]. The efficiency of antioxidant systems increases due to activation of the glutathione-ascorbate cycle and antioxidant enzymes [74,75]. Increased contents of glutathione are also related to improved metal sequestration via chelating and binding with phytochelatins [76,77]. Enhanced production of cysteine-rich compounds may also change the pattern of metal accumulation in plants, resulting in stabilization of toxic ions in the root system instead of their transport to photosynthetic parts [71]. Regulation of metal transport is associated with the $\mathrm{H}_{2} \mathrm{O}_{2}$ effect on the activity of vacuolar transmembrane proteins, such as ATPases [78]. Mechanisms of priming action of $\mathrm{H}_{2} \mathrm{O}_{2}$ are not limited to stimulation of the antioxidant system, but also include other cellular systems cooperating during stress. Among them is the glyoxalase system, composed of enzymes that detoxify methylglyoxal, a compound overproduced under environmental stress that is toxic at high concentrations [79]. Apart from a reduction in oxidative damage, rapeseed plants pretreated with $\mathrm{H}_{2} \mathrm{O}_{2}$ are capable of maintaining high activity of glyoxalases under $\mathrm{Cd}$ exposure. Another effect of $\mathrm{H}_{2} \mathrm{O}_{2}$-priming properties 
under metallic stress is balanced osmoregulation due to osmolyte accumulation. Enzymes of metabolic pathways of proline synthesis are upregulated after $\mathrm{H}_{2} \mathrm{O}_{2}$ pretreatment, whereas the activity of proline-degrading enzyme is reduced $[73,75,80]$.

Nitric oxide. Nitric oxide is a hydrophobic gaseous free radical, exhibiting both prooxidant and antioxidant properties [81], and exerting either cytotoxic or cytoprotective effects in plants. Therefore, its exploitation as a priming agent depends on the dose and the mode of application of NO-donor compounds [16]. Due to its gaseous nature, NO has to be applied to plants in the form of a donor compound. In studies concerning plant reaction to metallic stress, the most frequently used NO donor is sodium nitroprusside (SNP), but other substances are exploited as well (Table 2). In an experiment on rice subjected to Cd stress, $30 \mu \mathrm{M}$ S-nitroso- $N$-acetylpenicillamine (SNAP) functioned as a NO donor, increasing plant tolerance by improved antioxidant activity and plasma membrane structure [82]. In turn, it was found that SNP analogues: sodium nitrite and sodium ferrocyanide, unlike SNP, did not exhibit ameliorative activity under Cd stress [83]. Notwithstanding, [84] pointed out beneficial effects of NO donors such as potassium nitrite (PN), S-nitrosoglutathione (GSNO), diethylenetriamine/NO adduct (DETA/NO), SNAP, and S-nitroso-mercaptosuccinic acid (S-nitroso-MSA) on plants exposed to a wide range of environmental stresses.

Nitric oxide belongs to gasotransmitters-small molecules involved in plant physiology regulation [85]. Because of its versatile signaling role, NO is widely applied as a priming agent to enhance plant survival under abiotic stresses. [86] provided a comprehensive review of $\mathrm{NO}$ effects on plants treated with cadmium. They proposed that mechanisms of NO priming action involve protein modifications, activation of secondary messengers, including phytohormones and ROS, as well as alteration of protein kinase activities [86]. Survey of recent literature showed that the priming effect of NO and its interactions with other SSC are abundant in plants exposed to metallic (metalloid) stress, regardless of the toxic metal type [68,87-89] (Table 2). NO ameliorates plant growth as a result of reduced ROS production, minimization of oxidative damage, and improved antioxidant capacity [83,90-92]. Redox balance of the cell is maintained by an alternative redox-regulation system, composed of methionine sulfoxide reductases (MSR) [18]. Owing to the MSR capability of maintaining a proper redox state of methionine and preventing protein oxidation under environmental challenge, larger pools of glutathione and chaperones may be available for counteracting other stress effects in the cells [93]. Recent reports indicate that NO priming stabilizes redox status in plants exposed to toxic metals; thus, enhanced expression of MSR genes can be avoided [18]. In comparison with non-primed plants, NO-primed ones have a modified structure of cell walls and activity membrane transporters, and thus transportation of toxic ions is altered [68,82,94]. Photosynthetic performance is also improved, contributing to enhanced biomass accretion and proper organ development $[63,87]$.

Hydrogen sulfide. Similar to $\mathrm{NO}$, hydrogen sulfide $\left(\mathrm{H}_{2} \mathrm{~S}\right)$ is a gasotransmitter that can be either beneficial or toxic to plants. In millimolar concentrations it was considered phytotoxin for ages; however, in recent years its signaling role in plant organisms was ascertained. Studies revealed that the endogenous molecule is involved in numerous physiological processes, and a set of enzymes is available to regulate $\mathrm{H}_{2} \mathrm{~S}$ internal concentrations [95]. This compound interacts with other signaling molecules, mainly phytohormones and gasotransmitters, influences the functioning of ion channels and miRNA (micro RNA), thus indirectly regulating cell membrane polarization and transcription [67]. Priming action towards metallic stress is mainly attributed to enhanced antioxidant activity, particularly of the enzymes belonging to the AsA-GSH cycle [96], which contributes to reduced accumulation of ROS [89], improved metal chelation by GSH and phytochelatins [97,98], and diminished oxidative damage of macromolecules [99,100]. It also reduces nitroso-oxidative stress by interfering with NO- and ROS-associated metabolism [101]. $\mathrm{H}_{2} \mathrm{~S}$ interactions with $\mathrm{H}_{2} \mathrm{O}_{2}$ and $\mathrm{NO}$ are particularly reflected in changes in the activity of membrane transporters, affecting metal(loid) uptake and translocation [68,78]. Exogenous $\mathrm{H}_{2} \mathrm{~S}$ is applied in the form of donor compounds that degrade to release $\mathrm{H}_{2} \mathrm{~S}$ in the cells [102]. In plant systems, 
donor sulfide salts, mainly sodium hydrosulfide (NaHS) and sodium sulfide $\left(\mathrm{Na}_{2} \mathrm{~S}\right)$, are usually used (Table 2). Their solutions are easy to prepare and apply; however, release of gaseous $\mathrm{H}_{2} \mathrm{~S}$ to the atmosphere is quite fast. Recently, slow-release donor GYY-4137 (P-(4-methoxyphenyl)-P-4-morpholinyl-phosphinodithioic acid), which releases $\mathrm{H}_{2} \mathrm{~S}$ as a result of hydrolysis (and probably also in an enzymatic way) in a prolonged time period, was found to be more convenient to handle and experiment on plants [103]. There are no reports on its effect during metallic stress, but these are expected in the near future. An emerging area is the development of novel $\mathrm{H}_{2} \mathrm{~S}$ donors that are particularly important for medical purposes [102,104]. As these new drug formulations are developed, some of them may be applied in plant systems as prospective priming compounds (see the subsections below). The most important for broad agricultural exploitation is the low cost of a donor compound and the ease of simultaneous treatment of numerous seeds/plants [69].

Salicylic acid. Salicylic acid belongs to phenolic acids and is considered a plant growth regulator; thus, SA pretreatment is classified as hormopriming. Its mechanism of signaling and regulatory action, also involved in priming effects, is related predominantly to an interplay with a pool of other phytohormones and endogenous $\mathrm{H}_{2} \mathrm{O}_{2}$. SA is applied to prime plants against biotic stresses as it triggers the immune response to counteract pathogen attack [48] and against abiotic environmental stresses since it takes part in response development under osmotic and oxidative stress [105]. These "secondary stresses" are associated with salinity, ozone treatment, an exposure to toxic metals as well as pathogen attack $[48,106]$. Considering metallic stress, successful priming with SA was reported for several metals and in numerous plant species (Table 2). Beneficial effects were manifested mainly in an increased antioxidant capacity to scavenge ROS and other radicals [107-110] and in inhibited accumulation of toxic elements $[98,107,109,111,112]$. Pretreatment with SA prior to metallic stress affects the content and rearranges the profile of phenolic and organic acids [112], amino acids [98], as well as lipids and fatty acids [113,114]. A study on L. usitatissimum showed that the composition of plastidial membranes was preserved due to SA application prior to $\mathrm{Cd}$ treatment, which suggests the protection of the photosynthesis system from damage [113].

In light of recent findings, it seems that the plant capability of maintaining a relatively low content of endogenous SA and avoiding its elevation contribute to enhanced tolerance to metallic stress $[115,116]$. It is unresolved whether SA priming affects the biosynthesis pathway of endogenous SA and its final content. From a more practical point of view, studies evaluating the priming effect of SA derivatives and analogs, i.e., o-acetylosalicylic acid or 2,6-dichloroisonicotinic acid, are missing. These compounds enhance the plant response to other stresses, such as drought and salinity [14,106]; thus, it is substantiated to verify their suitability to induce trace metal tolerance as well.

From this compact review perspective, the similarities in response triggering may be used to elaborate a formulation for a "priming cocktail" that can be easily and at low cost applied in croplands to induce stress and even multistress tolerance. Regardless of their sole or joint application, small signaling compounds significantly contribute to increased survival and growth of plants in the presence of metallic elements. 


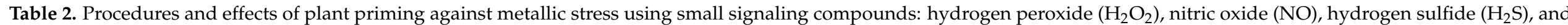
salicylic acid (SA).

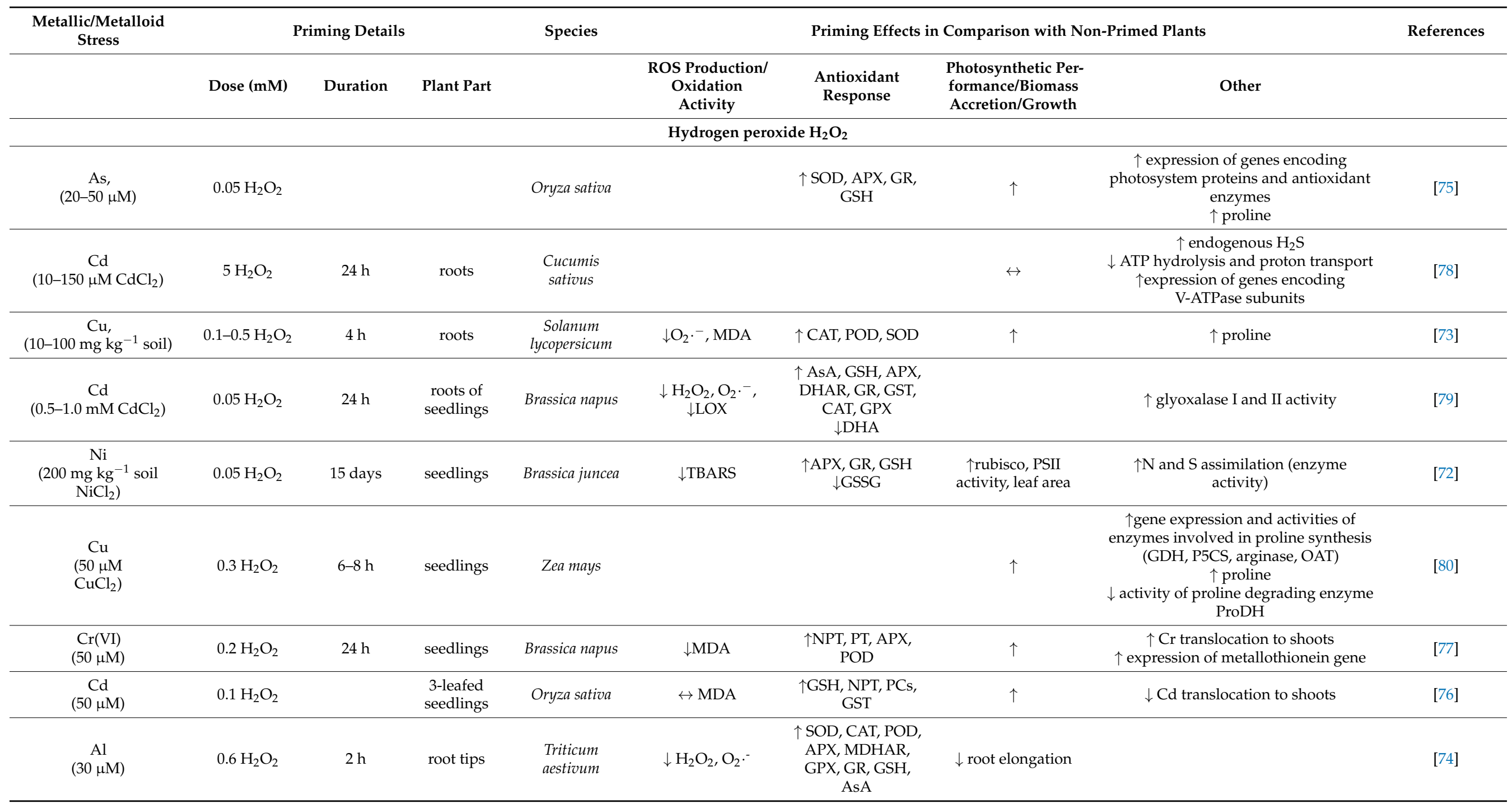


Table 2. Cont.

\begin{tabular}{|c|c|c|c|c|c|c|c|c|c|}
\hline \multirow{2}{*}{$\begin{array}{l}\text { Metallic/Metalloid } \\
\text { Stress }\end{array}$} & \multicolumn{3}{|c|}{ Priming Details } & \multirow[t]{2}{*}{ Species } & \multicolumn{4}{|c|}{ Priming Effects in Comparison with Non-Primed Plants } & \multirow[t]{2}{*}{ References } \\
\hline & Dose (mM) & Duration & Plant Part & & $\begin{array}{l}\text { ROS Production/ } \\
\text { Oxidation } \\
\text { Activity }\end{array}$ & $\begin{array}{l}\text { Antioxidant } \\
\text { Response }\end{array}$ & $\begin{array}{l}\text { Photosynthetic Per- } \\
\text { formance/Biomass } \\
\text { Accretion/Growth }\end{array}$ & Other & \\
\hline \multicolumn{10}{|c|}{ Nitric oxide NO } \\
\hline $\begin{array}{c}\mathrm{Cd} \\
\left(0.1 \mathrm{mM} \mathrm{CdCl}_{2}\right)\end{array}$ & $0.1 \mathrm{SNP}$ & 28 days & seedlings & $\begin{array}{l}\text { Triticum } \\
\text { aestivum }\end{array}$ & $\downarrow \mathrm{H}_{2} \mathrm{O}_{2}, \mathrm{MDA}$ & $\uparrow \mathrm{SOD}, \mathrm{CAT}, \mathrm{POD}$ & $\uparrow$ & $\begin{array}{c}\downarrow \downarrow \text { proline } \\
\downarrow \mathrm{Cd} \text { uptake and accumulation } \\
\uparrow \text { endogenous } \mathrm{H}_{2} \mathrm{~S} \\
\uparrow \mathrm{Zn}^{2+}, \mathrm{Fe}^{2+}, \mathrm{Ca}^{2+}, \mathrm{K}^{+} \text {content }\end{array}$ & [89] \\
\hline $\begin{array}{c}\mathrm{Cd} / \mathrm{Cu} \\
(0.2 \mathrm{mM} \text { each })\end{array}$ & $0.05 \mathrm{SNP}$ & 35 days & $\begin{array}{l}\text { in vitro } \\
\text { grown } \\
\text { plants }\end{array}$ & $\begin{array}{l}\text { Nicotiana } \\
\text { tabacum }\end{array}$ & & & $\begin{array}{l}\uparrow \text { rubisco and rubisco } \\
\text { activase content and } \\
\text { activity, depending } \\
\text { on the metal }\end{array}$ & & [63] \\
\hline $\begin{array}{c}\mathrm{Cd} \\
\left(10 \mu \mathrm{M} \mathrm{CdCl}_{2}\right)\end{array}$ & 0.03 SNAP & $2 \mathrm{~h}$ & $\begin{array}{l}\text { 3-leafed } \\
\text { seedlings }\end{array}$ & Oryza sativa & $\downarrow \mathrm{H}_{2} \mathrm{O}_{2}$ & $\begin{array}{c}\uparrow \mathrm{GSH}, \mathrm{APX}, \mathrm{SOD}, \\
\text { GR }\end{array}$ & $\uparrow$ & $\begin{array}{c}\text { affected abundance of plasma } \\
\text { membrane proteins (transporters, } \\
\text { ATPases, kinases, phosphatases, } \\
\text { phospholipases, enzymes, antiporters, } \\
\text { structural proteins, aquaporins, signal, } \\
\text { and hormone-related proteins) } \\
\text { 个phosphatidic acid }\end{array}$ & [82] \\
\hline $\begin{array}{c}\mathrm{Cd} \\
\left(100-200 \mu \mathrm{M} \mathrm{CdCl}_{2}\right)\end{array}$ & $\begin{array}{l}0.25 \\
\text { SNP }\end{array}$ & 14 days & seedlings & $\begin{array}{c}\text { Arachis } \\
\text { hypogaea }\end{array}$ & $\downarrow \mathrm{O}_{2} \cdot{ }^{-}, \mathrm{MDA}$ & $\begin{array}{c}\text { } \text { SOD, CAT, POD, } \\
\text { AsA }\end{array}$ & $\uparrow$ & $\begin{array}{c}\downarrow C d \text { translocation to shoots, } \\
\uparrow \text { enhanced Cd binding in cell walls } \\
\uparrow \text { proline }\end{array}$ & [92] \\
\hline $\begin{array}{c}\mathrm{Mn} \\
\left(1000 \mu \mathrm{M} \mathrm{MnCl}_{2}\right)\end{array}$ & $\begin{array}{l}0.1-1 \\
\text { SNP }\end{array}$ & 7 days & seeds & $\begin{array}{l}\text { Matricaria } \\
\text { chamomilla }\end{array}$ & $\downarrow$ ROS & $\begin{array}{l}\uparrow \mathrm{APX} \\
\downarrow \mathrm{CAT}\end{array}$ & $\uparrow$ & $\downarrow$ Mn content in roots and shoots & [91] \\
\hline $\begin{array}{c}\mathrm{Al} \\
\left.(100 \mu \mathrm{M} \mathrm{AlCl})_{3}\right)\end{array}$ & $0.01-0.05 \mathrm{SNP}$ & $12 \mathrm{~h}$ & seedlings & Glycine max & & & & $\begin{array}{c}\text { cooperates with } \mathrm{H}_{2} \mathrm{~S} \text { in induction of } \\
\text { citrate transporter expression } \\
\uparrow \text { endogenous } \mathrm{H}_{2} \mathrm{~S} \\
\uparrow \text { activities of } \mathrm{H}_{2} \mathrm{~S} \text { biosynthesis } \\
\text { enzymes (cysteine desulfhydrases, } \\
\text { CAS) } \\
\text { } \\
\text { activity of } \mathrm{H}_{2} \mathrm{~S}-\text { degrading enzyme } \\
\text { (OAS-TL) }\end{array}$ & [68] \\
\hline $\begin{array}{c}\mathrm{Pb} \\
\left(50 \mu \mathrm{M} \mathrm{Pb}\left(\mathrm{NO}_{3}\right)_{2}\right)\end{array}$ & $0.1 \mathrm{SNP}$ & $2-8 \mathrm{~h}$ & $\begin{array}{c}\text { germinating } \\
\text { seeds }\end{array}$ & $\begin{array}{l}\text { Triticum } \\
\text { aestivum }\end{array}$ & $\begin{array}{c}\downarrow \mathrm{MDA}, \\
\text { conjugated dienes, } \\
\mathrm{O}_{2} \cdot{ }^{-}, \cdot \mathrm{HO}\end{array}$ & $\begin{array}{l}\uparrow \mathrm{APX}, \mathrm{GPX}, \mathrm{GR}, \\
\text { SOD }\end{array}$ & $\begin{array}{c}\uparrow \text { radicle and } \\
\text { plumule length }\end{array}$ & $\uparrow$ intracellular nitrite content & [87] \\
\hline
\end{tabular}


Table 2. Cont.

\begin{tabular}{|c|c|c|c|c|c|c|c|c|c|}
\hline \multirow[t]{2}{*}{$\begin{array}{l}\text { Metallic/Metalloid } \\
\text { Stress }\end{array}$} & \multicolumn{3}{|c|}{ Priming Details } & \multirow[t]{2}{*}{ Species } & \multicolumn{4}{|c|}{ Priming Effects in Comparison with Non-Primed Plants } & \multirow[t]{2}{*}{ References } \\
\hline & Dose (mM) & Duration & Plant Part & & $\begin{array}{l}\text { ROS Production/ } \\
\text { Oxidation } \\
\text { Activity }\end{array}$ & $\begin{array}{l}\text { Antioxidant } \\
\text { Response }\end{array}$ & $\begin{array}{l}\text { Photosynthetic Per- } \\
\text { formance/Biomass } \\
\text { Accretion/Growth }\end{array}$ & Other & \\
\hline $\begin{array}{c}\mathrm{Cd} \\
(100 \mu \mathrm{M})\end{array}$ & $0.05 \mathrm{SNP}$ & & & $\begin{array}{l}\text { Trifolium } \\
\text { repens }\end{array}$ & $\downarrow \mathrm{H}_{2} \mathrm{O}_{2}, \mathrm{MDA}$ & $\begin{array}{l}\uparrow \text { ROS scavengers } \\
\text { (enzymatic and } \\
\text { non-enzymatic) }\end{array}$ & $\uparrow$ & $\begin{array}{c}\downarrow \text { inhibition of } \mathrm{H}^{+} \text {-ATPase proton } \\
\text { pumps } \\
\quad \uparrow \text { jasmonic acid, proline } \\
\downarrow \text { salicylic acid, ethylene } \\
\uparrow \mathrm{Mg}^{2+}, \mathrm{Cu}^{2+}, \mathrm{Ca}^{2+}, \mathrm{Fe}^{2+}\end{array}$ & [83] \\
\hline $\mathrm{Ni}$ & SNP & & & Oryza sativa & $\downarrow \mathrm{H}_{2} \mathrm{O}_{2}, \mathrm{MDA}$ & $\uparrow$ AsA, POD, CAT & $\uparrow$ & $\begin{array}{c}\uparrow \text { soluble proteins } \uparrow \text { proline } \uparrow \text { transcript } \\
\text { levels of CAT, POD, APX, GR, SOD } \\
\text { genes }\end{array}$ & {$[88]$} \\
\hline $\begin{array}{c}\text { As } \\
(0.25-0.5 \mathrm{mM} \\
\left.\mathrm{NaHAsO}_{4}\right)\end{array}$ & $0.25 \mathrm{SNP}$ & $72 \mathrm{~h}$ & seedlings & $\begin{array}{l}\text { Triticum } \\
\text { aestivum }\end{array}$ & $\downarrow \mathrm{H}_{2} \mathrm{O}_{2}, \mathrm{MDA}$ & $\begin{array}{c}\uparrow \text { AsA, GSH, } \\
\text { GSH/GSSG, } \\
\text { MDHAR, DHAR, } \\
\text { GR, GPX, CAT } \\
\downarrow \text { GSSG }\end{array}$ & & $\begin{array}{c}\uparrow \text { proline } \\
\uparrow \text { glyoxalase I and II activity }\end{array}$ & {$[90]$} \\
\hline $\begin{array}{c}\mathrm{Cd} \\
\left(100 \mu \mathrm{M} \mathrm{CdCl}_{2}\right) \\
\end{array}$ & $0.1 \mathrm{SNP}$ & 3 days & plants & $\begin{array}{c}\text { Arabidopsis } \\
\text { thaliana }\end{array}$ & $\begin{array}{c}\downarrow \text { protein oxidation } \\
\downarrow \text { ROS/peroxides } \\
\end{array}$ & $\uparrow \mathrm{GPX}, \mathrm{APX}, \mathrm{CAT}$ & & $\begin{array}{l}\downarrow \text { expression of methionine sulfoxide } \\
\text { reductase family genes }\end{array}$ & [18] \\
\hline $\begin{array}{c}\mathrm{Cd} \\
\left(0.1 \mathrm{mM} \mathrm{CdCl}_{2}\right)\end{array}$ & $0.2 \mathrm{NaHS}$ & 28 days & seedlings & $\begin{array}{l}\text { Triticum } \\
\text { aestivum }\end{array}$ & $\downarrow \mathrm{H}_{2} \mathrm{O}_{2}, \mathrm{MDA}$, & $\uparrow \mathrm{SOD}, \mathrm{CAT}, \mathrm{POD}$ & $\uparrow$ & $\begin{array}{c}\downarrow \downarrow \text { proline } \\
\downarrow \text { Cd content } \\
\uparrow \text { endogenous NO } \\
\uparrow \mathrm{Zn}^{2+}, \mathrm{Fe}^{2+}, \mathrm{Ca}^{2+}, \mathrm{K}^{+} \text {contents }\end{array}$ & [89] \\
\hline $\begin{array}{c}\mathrm{Al} \\
\left.(100 \mu \mathrm{M} \mathrm{AlCl})_{3}\right)\end{array}$ & $\begin{array}{l}0.01-0.1 \\
\text { NaHS }\end{array}$ & $12 \mathrm{~h}$ & seedlings & Glycine max & & & $\uparrow$ root growth & $\begin{array}{c}\downarrow \text { Al content in root tips } \\
\uparrow \text { citrate secretion } \\
\text { upregulation of plasma membrane } \\
\text { H+-ATPase (proton pump) } \\
\text { act downstream of NO activity in } \\
\text { Al-tolerance }\end{array}$ & {$[68]$} \\
\hline$\frac{\mathrm{Cd}}{\left(5-50 \mu \mathrm{M} \mathrm{CdCl}_{2}\right)}$ & $\begin{array}{l}0.2 \\
\mathrm{NaHS}\end{array}$ & $48 \mathrm{~h}$ & seedlings & $\begin{array}{l}\text { Hordeum } \\
\text { vulgare }\end{array}$ & $\begin{array}{c}\downarrow \mathrm{MDA}, \mathrm{H}_{2} \mathrm{O}_{2} \\
\mathrm{O}_{2} \cdot^{-}\end{array}$ & $\begin{array}{c}\uparrow \text { SOD, POD, APX } \\
\uparrow \text { GSH, AsA (at } \\
\text { moderate Cd } \\
\text { level) } \\
\downarrow \text { CAT }\end{array}$ & $\uparrow$ & $\downarrow C d$ content & [100] \\
\hline
\end{tabular}


Table 2. Cont.

\begin{tabular}{|c|c|c|c|c|c|c|c|c|c|}
\hline \multirow[t]{2}{*}{$\begin{array}{l}\text { Metallic/Metalloid } \\
\text { Stress }\end{array}$} & \multicolumn{3}{|c|}{ Priming Details } & \multirow[t]{2}{*}{ Species } & \multicolumn{4}{|c|}{ Priming Effects in Comparison with Non-Primed Plants } & \multirow[t]{2}{*}{ References } \\
\hline & Dose (mM) & Duration & Plant Part & & $\begin{array}{l}\text { ROS Production/ } \\
\text { Oxidation } \\
\text { Activity }\end{array}$ & $\begin{array}{l}\text { Antioxidant } \\
\text { Response }\end{array}$ & $\begin{array}{l}\text { Photosynthetic Per- } \\
\text { formance/Biomass } \\
\text { Accretion/Growth }\end{array}$ & Other & \\
\hline $\begin{array}{c}\mathrm{Cd} \\
(10-150 \mu \mathrm{M} \mathrm{CdCl} 2)\end{array}$ & $0.1 \mathrm{NaHS}$ & $24 \mathrm{~h}$ & roots & $\begin{array}{l}\text { Cucumis } \\
\text { sativus }\end{array}$ & & & $\uparrow$ & $\begin{array}{c}\uparrow \text { endogenous } \mathrm{H}_{2} \mathrm{~S} \\
\leftrightarrow \text { endogenous } \mathrm{H}_{2} \mathrm{O}_{2} \\
\uparrow \text { ATP hydrolysis and proton transport } \\
\uparrow \text { expression of genes encoding } \\
\text { V-ATPase subunits } \\
\uparrow \text { activity and transcripts of plasma } \\
\text { membrane NADPH oxidase }\end{array}$ & [78] \\
\hline $\begin{array}{c}\mathrm{Pb} \\
(100-400 \mu \mathrm{M} \\
\left.\mathrm{Pb}\left(\mathrm{NO}_{3}\right)_{2}\right) \\
\end{array}$ & $\begin{array}{l}0.1-0.2 \\
\mathrm{NaHS}\end{array}$ & $15 \mathrm{~d}$ & seedlings & Brassica napus & $\begin{array}{l}\downarrow \mathrm{MDA}, \mathrm{H}_{2} \mathrm{O}_{2}, \\
\mathrm{O}_{2} \cdot{ }^{-},-\mathrm{OH}\end{array}$ & $\begin{array}{l}\text { } \text { SOD, POD, APX, } \\
\text { CAT, GR, AsA, } \\
\text { GSH, GSSG }\end{array}$ & $\uparrow$ biomass & $\begin{array}{c}\downarrow \mathrm{Na}^{+} \text {uptake } \\
\uparrow \text { micro- and macroelement uptake } \\
\uparrow \text { total soluble proteins }\end{array}$ & [99] \\
\hline $\begin{array}{c}\mathrm{Pb} \\
\left(2.5 \mathrm{mM} \mathrm{Pb}\left(\mathrm{NO}_{3}\right)_{2}\right)\end{array}$ & $\begin{array}{l}0.2-2 \\
\mathrm{NaHS}\end{array}$ & $12 \mathrm{~h}$ & seeds & Zea mays & & $\uparrow \mathrm{GSH}$ & $\uparrow$ & $\begin{array}{c}\downarrow \text { Pb content } \\
\downarrow \text { amino acids: Asp, Glu, Asn, Ser, Hist, } \\
\text { Gly, Threo, Ala, Cyst } \\
\uparrow \text { amino acids: Tyr, Tryp } \\
\uparrow N R \text { activity } \\
\uparrow \text { protein content }\end{array}$ & [98] \\
\hline $\begin{array}{c}\mathrm{Cr} \\
\left(200 \mu \mathrm{M} \mathrm{K}_{2} \mathrm{Cr}_{2} \mathrm{O}_{7}\right)\end{array}$ & 0.5 & $9 \mathrm{~d}$ & seeds & Zea mays & $\downarrow \mathrm{H}_{2} \mathrm{O}_{2}$ & $\begin{array}{l}\stackrel{\leftrightarrow \text { GPOX }}{\downarrow \text { GST, SOD }} \\
\quad \text { GSNOR }\end{array}$ & $\uparrow$ radicle length & $\begin{array}{c}\downarrow \text { protein carbonylation and thiol } \\
\text { oxidation } \\
\uparrow \text { endogenous NO } \\
\downarrow \text { NADPH oxidase activity } \\
\uparrow \text { S-nitrosoglutathione }\end{array}$ & [101] \\
\hline \multicolumn{10}{|c|}{ Salicylic acid (SA) } \\
\hline $\begin{array}{c}\mathrm{Cd} \\
(0.25-0.5 \mathrm{mM} \\
\left.\mathrm{Cd}\left(\mathrm{NO}_{3}\right)_{2}\right)\end{array}$ & $0.5 \mathrm{SA}$ & $24 \mathrm{~h}$ & seeds & Silenesendtneri & & $\uparrow \mathrm{POD}$ & $\begin{array}{l}\text { } \text { germination and } \\
\text { seedling } \\
\text { development (root } \\
\text { length, shoot } \\
\text { biomass) }\end{array}$ & $\begin{array}{l}\uparrow \mathrm{Cd} \text { content in shoots } \\
\downarrow \text { Cd content in roots } \\
\text { altered profile of secondary metabolites: } \\
\text { phenolic compounds and organic acids }\end{array}$ & [112] \\
\hline $\begin{array}{c}\mathrm{Pb} \\
\left(1 \mathrm{mM} \mathrm{PbCl}_{2}\right)\end{array}$ & $100 \mathrm{mg} \cdot \mathrm{L}^{-1}$ & $24 \mathrm{~h}$ & seeds & Oryza sativa & $\begin{array}{l}\downarrow \mathrm{H}_{2} \mathrm{O}_{2}, \mathrm{O}_{2} \cdot{ }^{-} \\
-\mathrm{OH}, \mathrm{MDA}\end{array}$ & $\begin{array}{c}\uparrow \text { SOD, POD, CAT } \\
\uparrow G S H\end{array}$ & $\uparrow$ shoot growth & $\begin{array}{l}\downarrow \mathrm{Pb} \text { content in shoots } \\
\leftrightarrow \text { Pb content in roots } \\
\leftrightarrow \text { macronutrient uptake }\end{array}$ & [107] \\
\hline $\begin{array}{c}\mathrm{Ni} \\
\left(0.25 \mathrm{mM} \mathrm{NiSO}_{4}\right.\end{array}$ & $100 \mathrm{mg} \cdot \mathrm{L}^{-1}$ & $24 \mathrm{~h}$ & seeds & Oryza sativa & $\begin{array}{l}\downarrow \mathrm{H}_{2} \mathrm{O}_{2}, \mathrm{O}_{2} \cdot \cdot^{-} \\
-\mathrm{OH}, \mathrm{MDA} \\
\downarrow \mathrm{XOD}, \mathrm{MAO}\end{array}$ & $\begin{array}{l}\uparrow \text { CAT, GR, SOD, } \\
\text { GPX, POD } \\
\uparrow G S H, \text { AsA, Ve }\end{array}$ & $\uparrow$ shoot growth & $\begin{array}{l}\text { } \text { uptake of macronutrients } \\
\downarrow \text { Ni content in shoots } \\
\leftrightarrow \mathrm{Ni} \text { content in roots }\end{array}$ & [108] \\
\hline
\end{tabular}


Table 2. Cont.

\begin{tabular}{|c|c|c|c|c|c|c|c|c|c|}
\hline \multirow[t]{2}{*}{$\begin{array}{l}\text { Metallic/Metalloid } \\
\text { Stress }\end{array}$} & \multicolumn{3}{|c|}{ Priming Details } & \multirow[t]{2}{*}{ Species } & \multicolumn{4}{|c|}{ Priming Effects in Comparison with Non-Primed Plants } & \multirow[t]{2}{*}{ References } \\
\hline & Dose (mM) & Duration & Plant Part & & $\begin{array}{l}\text { ROS Production/ } \\
\text { Oxidation } \\
\text { Activity }\end{array}$ & $\begin{array}{l}\text { Antioxidant } \\
\text { Response }\end{array}$ & $\begin{array}{l}\text { Photosynthetic Per- } \\
\text { formance/Biomass } \\
\text { Accretion/Growth }\end{array}$ & Other & \\
\hline $\begin{array}{c}\mathrm{Pb} \\
\left(2.5 \mathrm{mM} \mathrm{Pb}\left(\mathrm{NO}_{3}\right)_{2}\right)\end{array}$ & $0.5 \mathrm{SA}$ & $12 \mathrm{~h}$ & seeds & Zea mays & & $\uparrow \mathrm{GSH}$ & $\uparrow$ & $\begin{array}{c}\downarrow \text { Pb content } \\
\downarrow \text { amino acids: Asp, Glu, Asn, Ser, Hist, } \\
\text { Gly, Threo, Ala, Ile } \\
\uparrow N R \text { activity } \\
\uparrow \text { protein content }\end{array}$ & [98] \\
\hline $\begin{array}{c}\mathrm{Cd} \\
\left(500-1000 \mu \mathrm{M} \mathrm{CdCl} l_{2}\right)\end{array}$ & $0.5 \mathrm{SA}$ & $12 \mathrm{~h}$ & seeds & $\begin{array}{l}\text { Triticum } \\
\text { aestivum }\end{array}$ & & $\uparrow \mathrm{SOD}, \mathrm{POD}, \mathrm{CAT}$ & $\uparrow$ biomass & $\begin{array}{c}\downarrow C d \text { content in the leaves and roots } \\
\uparrow \text { proline } \\
\text { altered leaf anatomy }\end{array}$ & [111] \\
\hline $\begin{array}{c}\mathrm{Cd} \\
(50-100 \mu \mathrm{M} \mathrm{CdCl})\end{array}$ & $0.25-1 \mathrm{SA}$ & $8 \mathrm{~h}$ & seeds & $\begin{array}{c}\text { Linum } \\
\text { usitatissimum }\end{array}$ & $\begin{array}{l}\downarrow \text { membrane } \\
\text { stability }\end{array}$ & & & $\begin{array}{l}\text { changed profile of membrane lipids, } \\
\text { preserved composition of plastidial } \\
\text { lipids }\end{array}$ & [113] \\
\hline $\begin{array}{c}\mathrm{Cd} \\
\left.(50-100 \mu \mathrm{M} \mathrm{CdCl})_{2}\right)\end{array}$ & $0.25-1.0 \mathrm{SA}$ & $8 \mathrm{~h}$ & seeds & $\begin{array}{c}\text { Linum } \\
\text { usitatissimum }\end{array}$ & $\downarrow \mathrm{MDA}$ & & $\begin{array}{l}\uparrow \text { biomass, } \\
\text { chlorophylls }\end{array}$ & $\begin{array}{c}\downarrow \text { Cd content } \\
\downarrow \text { carotenoids } \\
\uparrow \text { total lipid content, altered fatty acid } \\
\text { composition } \\
\text { altered nutrient distribution between } \\
\text { roots and shoots }\end{array}$ & [114] \\
\hline $\begin{array}{c}\mathrm{Hg} \\
(50 \mu \mathrm{M} \mathrm{HgCl} 2)\end{array}$ & $0.05 \mathrm{SA}$ & & $\begin{array}{l}2 \mathrm{w} \text {-old } \\
\text { transplants }\end{array}$ & $\begin{array}{l}\text { Melissa } \\
\text { offcinalis }\end{array}$ & $\downarrow \mathrm{MDA}$ & $\uparrow \mathrm{DPPH}, \mathrm{FRAP}$ & $\uparrow$ & $\begin{array}{c}\uparrow \text { proline } \\
\uparrow \text { phenolic compounds } \\
\uparrow \text { expression of chlorophyll synthase } \\
\text { and PAL }\end{array}$ & [110] \\
\hline $\begin{array}{c}\mathrm{As} \\
\left(50-100 \mu \mathrm{M} \mathrm{Na}_{3} \mathrm{AsO}_{4}\right)\end{array}$ & $0.5-1.0 \mathrm{SA}$ & $12 \mathrm{~h}$ & seeds & $\begin{array}{l}\text { Triticum } \\
\text { aestivum }\end{array}$ & $\downarrow \mathrm{MDA}, \mathrm{H}_{2} \mathrm{O}_{2}$ & $\begin{array}{l}\uparrow \mathrm{SOD}, \mathrm{POD} \\
\leftrightarrow \mathrm{CAT}, \mathrm{APX}\end{array}$ & $\begin{array}{l}\uparrow \text { photosynthetic } \\
\text { rate, chlorophylls }\end{array}$ & $\begin{array}{c}\downarrow \text { As content, also in grains } \\
\uparrow \text { soluble sugars } \\
\uparrow \text { soluble proteins } \\
\uparrow \text { proline }\end{array}$ & [109] \\
\hline $\begin{array}{l}\downarrow \text { decrease/reductio } \\
\text { DHAR-dehydroasc } \\
\text { peroxidase, GR-gli } \\
\text { monoamine oxidase, } \\
\text { lyase, OAT-ornithi } \\
\text { species, SOD—supe }\end{array}$ & $\begin{array}{l}\text { increase } / \mathrm{e} \\
\text { ate reductase } \\
\text { hione reduct } \\
\text { DA-malond } \\
\text { minotransfe } \\
\text { de dismutas }\end{array}$ & $\begin{array}{l}\text { cement, } \\
\text { PH-2,2 } \\
\text { GSH-glu } \\
\text { hyde, M } \\
\text { P5CS-c } \\
\text { ARS-th }\end{array}$ & $\begin{array}{l}\text { effect. Ab } \\
\text { enyl 1-picr } \\
\text { one (reduc } \\
\mathrm{R} \text {-monod } \\
\text { 1-pyrrolin } \\
\text { rbituric aci }\end{array}$ & $\begin{array}{l}\text { tions: APX } \\
\text { razyl radical } \\
\text { SNOR-S-n } \\
\text { oascorbate } \mathrm{r} \\
\text { boxylate syr } \\
\text { tive substan }\end{array}$ & $\begin{array}{l}\text { orbate peroxidas } \\
\text { P-ferric reducin } \\
\text { oglutathione redu } \\
\text { tase, NPT-nonpr } \\
\text { e, PCs-phytoche } \\
\text { le-vitamin E, V- }\end{array}$ & $\begin{array}{l}\text { SA-ascorbic aci } \\
\text { tioxidant power, } \\
\text { e, GSSG-glutath } \\
\text { thiols, NR-nitr } \\
\text { s, POD-peroxid } \\
\text { ase-vacuolar-tyr }\end{array}$ & $\begin{array}{l}\text { AS-cyanoalanine s } \\
\text { - glutamate dehyd } \\
\text { e (oxidized), GST-g } \\
\text { eductase, PAL-pher } \\
\text { ProDH-proline del } \\
\text { TPase, XOD_-xanth }\end{array}$ & $\begin{array}{l}\text { thase, CAT-catalase, DHA-dehydroas } \\
\text { enase, GPOX—glutathione peroxidase, G } \\
\text { athione S-transferase, LOX-lipooxygen } \\
\text { alanine amonia-lyase, OAS-TL-O-acetyl } \\
\text { trogenase, PT-protein thiols, ROS-reac } \\
\text { oxidase. }\end{array}$ & $\begin{array}{l}\text { orbic acid, } \\
\text { x guaiacol } \\
\text { e, MAO- } \\
\text { rine (thiol) } \\
\text { ve oxygen }\end{array}$ \\
\hline
\end{tabular}




\section{Other Potent Chemical Priming Agents}

Polyamines. Polyamines (PAs) are polycationic compounds with two or more amino groups. They occur ubiquitously in living organisms, and the most abundant are diamine putrescine (Put), triamine spermidine (Spd), and tetraamine spermine (Spm) [117]. These active PAs participate in multiple biological processes, from the cellular to the whole organism level, having regulating and signaling functions [118]. In plants under stress, their ameliorative role is attributed to support the radical scavenging system and antioxidant response, modulation of cation channels and membrane stabilization, regulation of phytohormone synthesis, and epigenetic modifications [119]. From the priming point of view, particularly interesting are relationships between polyamines and SSC. During PA catabolism, production of $\mathrm{H}_{2} \mathrm{O}_{2}$ occurs, and the pool of SA is affected [120]. Additionally, PAs are substrates for NO synthesis [85]. Exogenous treatment with PAs may therefore alter the levels of these SSC and contribute to intensified tolerance response to metallic stress. PAs also form conjugates with hydroxycinnamic acid that structurally resemble phytochelatins and are likely involved in metal chelation [121]. In priming experiments aimed at inducing metallic stress tolerance, usually micro-millimolar (0.1-1 mM) solutions of Put, Spr, and Spm are used for soaking seeds or for foliar spraying. PAs stimulate the antioxidant system and reduce metal accumulation and macromolecule damage, as shown in studies on plants stressed with $\mathrm{Cd}, \mathrm{Pb}$ [122], $\mathrm{Cr}$ [123], and $\mathrm{Co}$ [124].

Melatonin and its derivatives. The role of indoleamine melatonin ( $N$-acetyl-5-methoxytry ptamine) (Mel), an animal hormone, in plant functioning was repeatedly reported in recent years $[125,126]$ when improved detection methods allowed precise determination of its content in plant tissues. It became apparent that both Mel and its metabolite, 2-hydroxymelatonin (2-OHMel), are capable of inducing tolerance responses in stressexposed plants and therefore are now exploited as chemical priming agents for chilling, salinity, and drought $[127,128]$. They are also efficient priming agents for combating metallic stress. Ameliorative action under metal exposure is mainly attributed to the modulation of antioxidant responses, enhanced accumulation of phenolics, carbohydrates, polyamines, and proteins, as well as to restricted metal translocation [129-133]. It has been recently shown that Mel protects cells from DNA damage and subsequent cell death induced by $\mathrm{Pb}$ [51].

Beneficial elements. Another strategy to increase toxic metal tolerance is the application of beneficial elements as priming agents, sometimes referred to as nutripriming. Solutions of numerous compounds are usually inexpensive and easy to handle and therefore, large amounts of seeds/seedlings can be rapidly primed. Recent reports show that encouraging results have been obtained in plant priming with silicon (Si) and selenium (Se) compounds. $\mathrm{Si}$, applied as $10^{-5} \mathrm{M}$ sodium metasilicate, ameliorated growth and the physiological response of Brassica juncea under Ni exposure by stimulation of antioxidant machinery [134]. In Capsicum annuum subjected to Cd stress, $\mathrm{Si}$ applied as $2 \mathrm{mM}$ sodium silicate, apart from stimulation of antioxidant system activity, promoted the accumulation of endogenous $\mathrm{H}_{2} \mathrm{O}_{2}$ and $\mathrm{NO}$ [135]. Enhanced synthesis of another SSC, $\mathrm{H}_{2} \mathrm{~S}$, occurred in zucchini plants primed with $\mathrm{Ca}^{+2}$ ions prior to Ni exposure [97]. Rice seedlings primed with $60 \mu \mathrm{M}$ Se had a more efficient antioxidant system and performed better under nutrient imbalances caused by $\mathrm{Pb}$ and $\mathrm{Ni}[107,108]$. Moreover, translocation of toxic ions can be significantly restricted as a result of Se priming [136]. Inevitably, an enormous potential for elemental priming against metallic stress is represented by nanoparticles. Their application causes significant improvements in antioxidant responses under metallic stress, as in the case of sunflower seeds primed with S nanoparticles prior to $\mathrm{Mn}$ treatment [137]. Se nanoparticles also have potential, as foliar spraying with this form of Se facilitated the synthesis of non-enzymatic antioxidants and enhanced the activity of antioxidant enzymes in coriander plants exposed to $\mathrm{Pb}$ stress [138]. A recent study revealed that Se nanoparticle application influenced not only the antioxidant system but also altered plant morphology and metabolite synthesis and contributed to the accumulation of epigenetic changes in DNA [139]. Such a versatile 
modulation of plant metabolism may facilitate the development of metallic stress tolerance as well.

\section{Genes That Can Be Targeted to Induce Priming Effects for Metallic Stress}

Genetic control of the priming phenomenon involves the regulation of numerous pathways activated during pre-treatment and subsequent stress events. New information on its mechanisms are gathered due to "omics" analyses that decipher changes in the transcriptome, proteome, and metabolome of primed plants [44,140-142]. However, the details of genetic control during priming against metallic stress are not fully elucidated. The recognition of potential genes that can be targeted may facilitate future studies on priming effectiveness towards tolerance to metal toxicity. Recent studies confirm that priming agents are potent activators of the genetic regulatory network and contribute to diversified expression of genes encoding stress-defense compounds in plants cultivated in contaminated soil [44]. The genes can be classified as those that control development and common versatile defense responses in plants, irrespective of the stress type and genes that are specifically expressed in the presence of toxic metals. The first group would include genes encoding (i) enzymes involved in antioxidant response pathways- the whole set of radical scavenging enzymes, including proteins of the ascorbate-glutathione cycle, as well as the enzymes of supportive redox systems (glyoxalase system, methionine sulfoxide reductases) [18,79], (ii) proteins involved in SSC production, (iii) enzymes crucial for PA and Mel biosynthesis pathways [143,144], (iv) heat shock proteins and other chaperones responsible for proper folding and (de)aggregation of proteins [145], (v) DNA repair enzymes [44], and (vi) proteins regulating developmental processes, such as stress-responsive asparagine rich protein (NRP) [146], signal transducing mitogen-activated kinases (MPK) or proteins involved in chromatin modifications [141]. As depicted in the subsections above and in Table 2, the priming agents predominantly affect the antioxidant activity, and numerous studies confirmed that enhanced expression of genes encoding antioxidant enzymes, superoxide dismutase (SOD), peroxidases (POD), and catalase (CAT), enzymes of the ascorbate-glutathione cycle, occurs as a priming effect $[44,75,88]$. Additionally, SOD and, in the presence of a strong reductant, also peroxidases take part in $\mathrm{H}_{2} \mathrm{O}_{2}$ production in numerous cell compartments [71]. Among the genes encoding enzymatic machinery for the synthesis of other SSC, interest should be paid to cysteine desulfhydrolase (CAS) (catalyzing $\mathrm{H}_{2} \mathrm{~S}$ formation) [68], the large gene family of nitric oxide synthases (NOS) (catalyzing NO synthesis) [147], as well as isochorismate synthase (ICS) and phenylalanine ammonia-lyase (PAL) involved in SA biosynthesis [148].

Putrescine and spermine are PAs particularly involved in stress tolerance (Put) and stress response (Spm) [117]. There is evidence that under stress, biosynthesis of endogenous Put occurs more frequently via an indirect route from arginine (in the arginine decarboxylase pathway ADC) rather than directly from ornithine (in the ornithine decarboxylase pathway ODC) [121,149], which suggests that the genes encoding proteins active in the ADC pathway should be chosen as prospective modification targets. In order to increase Spm accumulation, the activity/expression of the key rate-limiting enzyme, S-adenosylmethionine decarboxylase (SAMD), could be manipulated [143]. In the case of Mel, its biosynthesis may be promoted when a gene encoding the enzyme serotonin $\mathrm{N}$-acetyltransferase (SNAT) is overexpressed [144].

Plant cells are equipped with numerous stress- and damage-protective proteins whose expression and functioning can be manipulated to achieve priming effects. Heat shock proteins (HSPs) are involved in the response to environmental factors in almost all living organisms. Genes encoding plant HSPs are localized in nuclear and cytoplasmatic genomes, whereas proteins are abundant in various cell compartments. Diverse HSP variants detected in plants probably reflect molecular modifications forced by various stress conditions [150]. Although HSPs function in a wide range of abiotic stresses, including oxidative stress, the understanding of their specific roles in plants cells is still limited [151]. The involvement of mitochondrial sHSP23 and cytosolic sHSP17.7 proteins in As (as a met- 
alloid stress) and $\mathrm{Pb}$ tolerance has been reported in plants and bacteria [152-154], whereas expression of maize root HSP70 was upregulated by cesium oxide nanoparticles [155].

Protective functions also include proteins responsible for DNA repair since genome stability is essential to maintain growth and development [156]. The enzymes 8-oxoguanine glycosylase (OGG1) and formamidopyrimidine-DNA glycosylase (FPG) act through the base excision repair pathway, cutting off DNA bases that were irreversibly oxidized during stress exposure. Expression of genes encoding OGG1 and FPG was upregulated by hydroand biopriming, applied to improve plant performance during germination and growth in metal-contaminated soil [44]. It is noteworthy that the expression of DNA repair enzymes is particularly critical during seed imbibition and germination [156], the predominant stages in which priming is applied.

To the second group belong genes encoding (i) metal transporters, particularly vacuolar, such as ABC (ATP-binding cassette) proteins [78], CDF (cation diffusion facilitator), but also cytoplasmatic (ZIP-zinc/iron transport proteins: ZRT/IRT-related proteins) [157]. Included here are also (ii) genes encoding metal-binding peptides: metallothioneins (MTs) and enzymes responsible for the synthesis and transport of metal chelators: phytochelatins and low-molecular-weight organic acids (LMWOA). Phytochelatin synthase (PCS) is considered a crucial enzyme for metal tolerance and plant capability of phytoremediation [158]. In the case of LMWOA, the main role in acquiring metal(loid) tolerance is played by organic acid anion transporters belonging to the MATE (multidrug and toxic compound extrusion) family [68]. Products of their expression actively take part in metal detoxification by restricting or facilitating their translocation between plant organs and by exclusion of toxic ions from the cytoplasm and their sequestration in the vacuole. Additionally, MTs may function as DNA-repair compounds and therefore, their priming-related action may be more versatile [43]. Expression of MT-2 genes is particularly influenced during cell response to oxidative stress [44].

\section{Hydropriming, Halopriming, Hormopriming, and Biopriming}

These priming strategies involve the application of water, salt solution, phytohormones, and promoting microorganisms as priming agents. Suitability of hormopriming to achieve metallic stress tolerance and the protective mechanisms of particular phytohormones were recently comprehensively discussed in [50]. Here, this aspect will not be extensively presented since the mechanisms of action and impact of salicylic acid, the most frequently used priming agent among growth regulators, were described in the subsection above. However, all types of phytohormones, both growth promoters (auxins AUX, cytokinins CYT, gibberellins GAs) and inhibitors/regulators (abscisic acid ABA, jasmonic acid JA), as well as brassinosteroids (BR) and strigolactones (SL) are potent priming agents. On a molecular level, their stress-preventing action is attributed to signaling via phytohormonal cross-talks and interactions with the SSC synthesis pathways [49], regulation of gene expression, and influencing post-transcriptional protein modifications, as well as epigenetic control of stress-related genes $[159,160]$. Phytohormones are applied to plants via seed soaking, foliar spraying, and medium enrichment, with micromolar concentrations exerting a positive impact on plant functioning under metal exposure. Besides SA application, priming with AUX is the most frequent approach in combating metallic stress. Indole-3-acetic acid (IAA) and indole-3-butyric acid (IBA), applied in concentrations ranging from 2-500 $\mu \mathrm{M}$, ameliorate the toxicity of cadmium [111,161-165], copper [124,166,167], and aluminum [168]. Numerous studies report the beneficial activity of low BR doses (0.5-10 $\mu \mathrm{M})$ (24-epibrassinolide, 3-epibrassinolide) towards stress induced by metals and metalloids: As, Cd, Cr, and $\mathrm{Cu}[109,123,169,170]$. GAs $(1-100 \mu \mathrm{M})$ are effective priming agents diminishing the growth deteriorations caused by $\mathrm{Cu}$ and $\mathrm{Zn}[167,171]$. CYT (i.e., 10-100 $\mu \mathrm{M}$ 6-benzylaminopurine) reduced the detrimental effects of $\mathrm{Cd}$ and $\mathrm{Zn}$ toxicity [172,173], whereas jasmonic acid $(0.1 \mu \mathrm{M} \mathrm{JA})$ - ameliorated reaction to Pb stress [174]. Recently $1.0 \mu \mathrm{M} \mu \mathrm{M}$ SL analogue GR24 was found to combat Cd toxicity in switchgrass 
(Panicum virgatum) by altering nutrient and ballast metal uptake and interacting with the pool of endogenous strigolactones [175].

Halopriming involves salt treatments applied prior to exposure to stress conditions. It is commonly used in the form of cis-priming to induce tolerance to salinity and other osmotic stresses [176]. Recently, it was revealed that 10-h priming of wheat seeds with $7.5 \mathrm{mM}$ $\mathrm{Mg}\left(\mathrm{NO}_{3}\right)_{2}$ and $\left.\mathrm{Ca}\left(\mathrm{NO}_{3}\right)_{2}\right)$ improved seed germination under $\mathrm{Hg}$ application by stimulation of growth of seedling organs and carbohydrate metabolism [177]. Similar effectswere achieved by hydropriming, which consists of seed soaking in distilled water, without any other components [177]. An inevitable advantage of hydropriming is its simplicity and low cost, as well as the possibility to achieve a large population of primed plants. It has been long applied in agricultural production to synchronize seed germination and to improve yield [46]. Apart from improving germination characteristics, hydropriming also affects the expression of genes involved in antioxidant defense and metal chelation, as revealed in a Medicago truncatula grown in contaminated soil [44]. However, in numerous studies analyzing priming effects towards metallic stress tolerance, hydropriming is regarded as a control and as a reference treatment for proper assessment of the impact of priming compounds suspended in water solutions.

Biological priming, namely, priming by colonization of plants with beneficial microorganisms to induce stress tolerance, is gaining interest worldwide since it fits in and complements sustainable and organic plant cultivation. Beneficial microorganisms can be isolated and selected from heavy metal-polluted soils, particularly from the rhizosphere of metal-resistant plants, but also from aboveground organs. Bacterial species with plant growth-promoting activity belong to numerous genera, i.e., Alcaligenes, Curtobacterium, Bacillus, Microbacterium, Paenibacillus, Pseudomonas, Streptomyces, and Tetrathiobacter [178-180], whereas among fungi are rhizosphere endophytes, such as Piriformospora indica, Acrocalymma vagum, and Scytalidium lignicola [181,182]. Improved plant growth under metallic stress is attributed to bacterial capability of nitrogen assimilation, producing and secreting plant growth regulators, particularly IAA, siderophores, and various volatile organic compounds [183-185]. Fungal species stimulate the antioxidant system in plants and prevent cell death, as well as ameliorate water uptake, facilitating access to less available nutrient elements. Toxic metals can be solubilized or adsorbed on microorganism biomass, restricting their bioavailability and uptake by plants [186]. At the regulatory level, biopriming interferes with the expression of genes involved in stress responses [44,187], as well as with phytohormonal regulatory network [184].

\section{Effect-Oriented Priming: Growth Improvement, Metal Uptake Restriction or Metal Uptake Stimulation?}

Considering plant cultivation under exposure of metallic elements, the desired goals of priming can be directed either to improve seed germination, survival, and biomass accretion or to reduce uptake and transport of toxic ions in plant organs. Obviously, all priming agents are applied to achieve improved plant growth and yield, but some of them are also particularly effective in restricting metal accumulation. Analysis of available reports shows that the highest potential for decreasing metal accumulation in plant organs has chemical priming with the use of $\mathrm{NO}$ and $\mathrm{H}_{2} \mathrm{~S}$ donors, as well as with triamine-spermidine (Figure 1). $\mathrm{NO}$ and $\mathrm{H}_{2} \mathrm{~S}$ affect plasma membrane proteins, mainly transporters and antiporters $[68,78,82]$, whereas spermidine and its complexes take part in metal chelation and influence metal transportation within plant tissues $[122,123]$. The metal content in biomass can be also reduced by priming with PGRs with growth-inhibiting properties: JA, brassinosteroids, and strigolactones [169,174,175]. Among other techniques, the use of biopriming with selected bacterial bioremediator strains allows achieving a significant decrease in toxic metals accumulated in the plant yield $[179,183,185]$. There are also reports indicating that nutripriming with Se is effective in reducing the as content in the grains and thus restricts its circulation in the food chain [136]. 


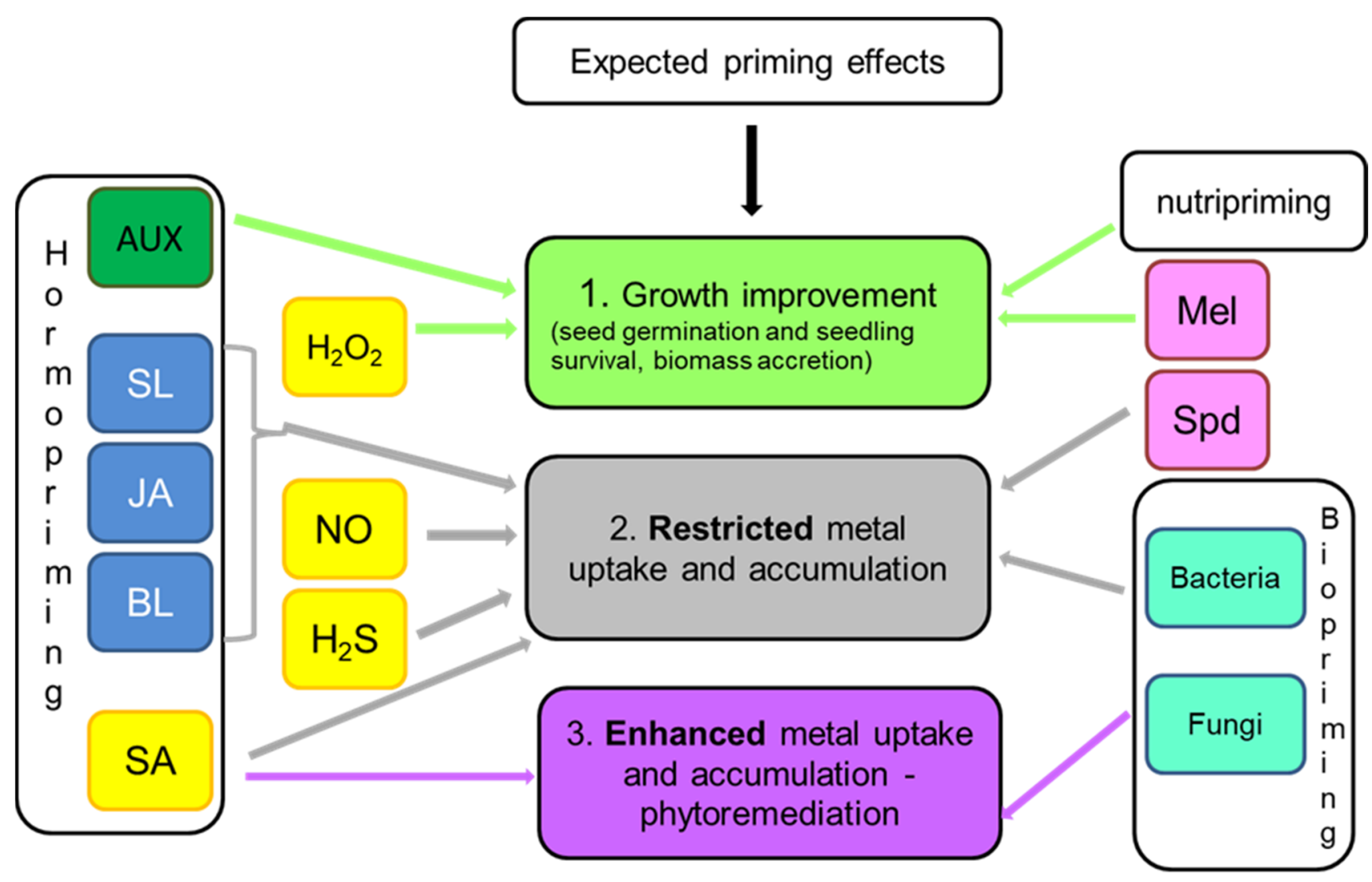

Figure 1. Effect-oriented priming — the choice of priming agent depends on the expected outcome of the priming procedure.Small signaling compounds (SSC) were colored yellow. Abbreviations: AUX-auxins, BL—brassinosteroids, JA—jasmonic acid, Mel—melatonin, SA—salicylic acid, SL—strigolactones, Spd—spermidine.

Priming techniques aimed at improving plant growth and physiological performance involve priming with $\mathrm{H}_{2} \mathrm{O}_{2}$ that stimulates antioxidant defense $[75,79,80]$ and hormopriming with PGRs of growth-promoting activities, particularly with AUX [162-164]. Application of melatonin, which is an indole compound sharing some routes of the biosynthetic pathway with AUX, also facilitates plant survival and biomass accretion under metallic stress [131-133] (Figure 1).

Another option is to use priming agents for stimulating metal removal from contaminated soil, facilitating its clean-up by phytoremediation. In such a case, a priming compound is expected to enhance metal uptake and accumulation by plants. Such effects can be obtained by priming with SA since this molecule differentially influences metal distribution between roots and shoots [107,112] (Figure 1). This feature can be used to manipulate a plant's ability to phytostabilize (in the root system) or phytoextract (in the aboveground biomass) metals. Significant improvement in metal uptake from the soil is also obtained by biopriming with fungal endophytes [182].

\section{Prospective Priming Approaches and Concluding Remarks}

Chemical priming gives an opportunity to elaborate "priming cocktails", mixtures of priming agents of which joint action would contribute to further enhancement of plant growth and productivity under unfavorable conditions. A multiple priming approach seems crucial if negative effects of multistress, encountered by plants under natural conditions, are to be diminished. Application of priming cocktails may also facilitate organic and sustainable crop production and reduce the amounts of agrochemicals [16]. A successful and prospective example of a versatile priming agent is NOSH-aspirin, a compound that acts as a donor of $\mathrm{NO}, \mathrm{H}_{2} \mathrm{~S}$, and the SA derivative acetylosalicylic acid. Priming with $\mathrm{NOSH}$-aspirin ameliorates the drought stress reaction of alfalfa plants by inducing typical 
defense reactions, particularly antioxidant capacity [14]. This novel compound is currently tested by patent owners in other plant-stress systems, including heavy metal exposure (communicated by Prof. K. Kashfi).

To combat metallic stress via priming, new priming agents and techniques are constantly developed and incorporated into practice. Some prospective multiple priming agents can be found among compounds applied in the case of other stresses. For instance, apoplastic fructans, flavonoids or amino acids (i.e., $\beta$-aminobutyric acid, vitamin U- Smethylmethionine) are capable of priming plant innate immunity and increasing cold tolerance $[12,188,189]$, but their stress-protective properties can be verified under metallic stress as well. One prospective is the exploitation of nanoparticles that induce significant variations in plant biochemistry, gene expression, and epigenetic DNA patterns [139], contributing to the development of altered (and probably more efficient) stress responses. Among physical priming methods, magnetopriming was reported to stimulate antioxidant system activity under salt stress [190], and therefore this technique may have significant potential for metallic stress amelioration. Finally, natural organic extracts can also be sources of priming agents or constitute priming cocktails themselves, as shown by a recent study on the amelioration of $\mathrm{Pb}$ toxicity by seed priming with weed extracts [191].

In conclusion, all priming strategies towards improved plant tolerance to toxic metal and metalloids have important features in common: Are easy to apply to numerous plants, are not labor-consuming, do not require sophisticated laboratory equipment, and are relatively inexpensive. For these reasons, priming can be routinely applied in agricultural and horticultural practice all over the world. These are inevitable advantages over stress tolerance induction via modern genetic engineering techniques, and therefore novel and improved priming methods are expected to constantly develop. The joint knowledge gathered here, covering the biochemical and genetic background of the priming phenomenon, may facilitate designing and conducting future research for plant tolerance to metal and metalloid stress.

Supplementary Materials: The following are available online at https:/ /www.mdpi.com/2223-774 7/10/4/623/s1, Table S1: Application of metals as priming agents towards secondary non-metallic stress tolerance.

Funding: This research was funded by Ministry of Science and Higher Education of the Republic of Poland, grant number 0050012-D011.

Institutional Review Board Statement: Not applicable.

Informed Consent Statement: Not applicable.

Data Availability Statement: Not applicable.

Conflicts of Interest: The author declares no conflict of interest.

\section{References}

1. Wu, Q.; Leung, J.Y.; Geng, X.; Chen, S.; Huang, X.; Li, H.; Huang, Z.; Zhu, L.; Chen, J.; Lu, Y. Heavy metal contamination of soil and water in the vicinity of an abandoned e-waste recycling site: Implications for dissemination of heavy metals. Sci. Total. Environ. 2015, 506-507, 217-225. [CrossRef] [PubMed]

2. Singh, S.; Parihar, P.; Singh, R.; Singh, V.P.; Prasad, S.M. Heavy metal tolerance in plants: Role of transcriptomics, proteomics, metabolomics, and ionomics. Front. Plant Sci. 2016, 6, 1143. [CrossRef]

3. Hasan, K.; Cheng, Y.; Kanwar, M.K.; Chu, X.-Y.; Ahammed, G.J.; Qi, Z.-Y. Responses of plant proteins to heavy metal stress-A review. Front. Plant Sci. 2017, 8, 1492. [CrossRef] [PubMed]

4. Wójcik, M.; Tukiendorf, A. Accumulation and tolerance of lead in two contrasting ecotypes of Dianthus carthusianorum. Phytochemistry 2014, 100, 60-65. [CrossRef]

5. Muszyńska, E.; Labudda, M.; Hanus-Fajerska, E. Changes in proteolytic activity and protein carbonylation in shoots of Alyssum montanum ecotypes under multi-metal stress. J. Plant Physiol. 2019, 232, 61-64. [CrossRef] [PubMed]

6. Wiszniewska, A.; Kamińska, I.; Hanus-Fajerska, E.; Sliwinska, E.; Koźmińska, A. Distinct co-tolerance responses to combined salinity and cadmium exposure in metallicolous and non-metallicolous ecotypes of Silene vulgaris. Ecotoxicol. Environ. Saf. 2020, 201, 110823. [CrossRef] 
7. Cabello-Conejo, M.; Prieto-Fernandez, A.; Kidd, P. Exogenous treatments with phytohormones can improve growth and nickel yield of hyperaccumulating plants. Sci. Total. Environ. 2014, 494-495, 1-8. [CrossRef]

8. Rees, F.; Sterckeman, T.; Morel, J.L. Root development of non-accumulating and hyperaccumulating plants in metal-contaminated soils amended with biochar. Chemosphere 2016, 142, 48-55. [CrossRef]

9. Moya, J.L.; Ros, R.; Picazo, I. Heavy metal-hormone interactions in rice plants: Effects on growth, net photosynthesis, and carbohydrate distribution. J. Plant Growth Regul. 1995, 14, 61-67. [CrossRef]

10. Shahid, M.; Pourrut, B.; Dumat, C.; Nadeem, M.; Aslam, M.; Pinelli, E. Heavy-Metal-Induced Reactive Oxygen Species: Phytotoxicity and Physicochemical Changes in Plants; Springer: Berlin/Heidelberg, Germany, 2014; pp. 1-44.

11. Sruthi, P.; Shackira, A.M.; Puthur, J.T. Heavy metal detoxification mechanisms in halophytes: An overview. Wetl. Ecol. Manag. 2017, 25, 129-148. [CrossRef]

12. Balmer, A.; Pastor, V.; Gamir, J.; Flors, V.; Mauch-Mani, B. The 'prime-ome': Towards a holistic approach to priming. Trends Plant Sci. 2015, 20, 443-452. [CrossRef] [PubMed]

13. Vincent, C.; Rowland, D.; Schaffer, B.; Bassil, E.; Racette, K.; Zurweller, B. Primed acclimation: A physiological process offers a strategy for more resilient and irrigation-efficient crop production. Plant Sci. 2020, 295, 110240. [CrossRef] [PubMed]

14. Antoniou, C.; Xenofontos, R.; Chatzimichail, G.; Christou, A.; Kashfi, K.; Fotopoulos, V. Exploring the potential of nitric oxide and hydrogen sulfide (NOSH)-releasing synthetic compounds as novel priming agents against drought stress in Medicago sativa plants. Biomolecules 2020, 10, 120. [CrossRef]

15. Leuendorf, J.E.; Frank, M.; Schmülling, T. Acclimation, priming and memory in the response of Arabidopsis thaliana seedlings to cold stress. Sci. Rep. 2020, 10, 1-11. [CrossRef] [PubMed]

16. Savvides, A.; Ali, S.; Tester, M.; Fotopoulos, V. Chemical priming of plants against multiple abiotic stresses: Mission possible? Trends Plant Sci. 2016, 21, 329-340. [CrossRef]

17. Filippou, P.; Tanou, G.; Molassiotis, A.; Fotopoulos, V. Plant Acclimation to Environmental Stress Using Priming Agents; Springer: Berlin/Heidelberg, Germany, 2012; pp. 1-27.

18. Méndez, A.A.; Pena, L.B.; Benavides, M.P.; Gallego, S.M. Priming with NO controls redox state and prevents cadmium-induced general up-regulation of methionine sulfoxide reductase gene family in Arabidopsis. Biochimie 2016, 131, 128-136. [CrossRef]

19. Wiszniewska, A.; Muszyńska, E.; Kołton, A.; Kamińska, I.; Hanus-Fajerska, E. In vitro acclimation to prolonged metallic stress is associated with modulation of antioxidant responses in a woody shrub Daphne jasminea. Plant Cell. Tissue Organ Cult. 2019, 139, 339-357. [CrossRef]

20. Hilker, M.; Schwachtje, J.; Baier, M.; Balazadeh, S.; Bäurle, I.; Geiselhardt, S.; Dirk, K.H.; Reinhard, K.; Bernd, M.-R.; Matthias, C.R.; et al. Priming and memory of stress responses in organisms lacking a nervous system. Biol. Rev. 2016, 91, 1118-1133. [CrossRef]

21. Antoniou, C.; Savvides, A.; Christou, A.; Fotopoulos, V. Unravelling chemical priming machinery in plants: The role of reactive oxygen-nitrogen-sulfur species in abiotic stress tolerance enhancement. Curr. Opin. Plant Biol. 2016, 33, 101-107. [CrossRef]

22. González-Bosch, C. Priming plant resistance by activation of redox-sensitive genes. Free. Radic. Biol. Med. 2018, 122, 171-180. [CrossRef]

23. Rowland, D.L.; Faircloth, W.H.; Payton, P.; Tissue, D.T.; Ferrell, J.A.; Sorensen, R.B.; Butts, C.L. Primed acclimation of cultivated peanut (Arachis hypogaea L.) through the use of deficit irrigation timed to crop developmental periods. Agric. Water Manag. 2012, 113, 85-95. [CrossRef]

24. Poschenrieder, C.; Cabot, C.; Martos, S.; Gallego, B.; Barceló, J. Do toxic ions induce hormesis in plants? Plant Sci. 2013, 212, 15-25. [CrossRef] [PubMed]

25. Wiszniewska, A.; Hanus-Fajerska, E.; Muszyńska, E.; Smoleń, S. Comparative assessment of response to cadmium in heavy metal-tolerant shrubs cultured in vitro. Water Air Soil Pollut. 2017, 228, 1-13. [CrossRef] [PubMed]

26. Wiszniewska, A.; Muszyńska, E.; Hanus-Fajerska, E.; Dziurka, K.; Dziurka, M. Evaluation of the protective role of exogenous growth regulators against Ni toxicity in woody shrub Daphne jasminea. Planta 2018, 248, 1365-1381. [CrossRef]

27. Calabrese, E.J.; Blain, R.B. Hormesis and plant biology. Environ. Pollut. 2009, 157, 42-48. [CrossRef]

28. Calabrese, E.J.; Baldwin, L.A. The hormetic dose-response model is more common than the threshold model in toxicology. Toxicol. Sci. 2003, 71, 246-250. [CrossRef]

29. Sivritepe, N.; Sivritepe, H.; Eris, A. The effects of $\mathrm{NaCl}$ priming on salt tolerance in melon seedlings grown under saline conditions. Sci. Hortic. 2003, 97, 229-237. [CrossRef]

30. Hassini, I.; Martinez-Ballesta, M.C.; Boughanmi, N.; Moreno, D.A.; Carvajal, M. Improvement of broccoli sprouts (Brassica oleracea L. var. italica) growth and quality by $\mathrm{KCl}$ seed priming and methyl jasmonate under salinity stress. Sci. Hortic. 2017, 226, 141-151. [CrossRef]

31. Zhao, W.; Dong, H.; Zhou, Z.; Wang, Y.; Hu, W. Potassium (K) application alleviates the negative effect of drought on cotton fiber strength by sustaining higher sucrose content and carbohydrates conversion rate. Plant Physiol. Biochem. 2020, 157, 105-113. [CrossRef]

32. Van Nguyen, D.; Nguyen, H.M.; Le, N.T.; Nguyen, K.H.; Nguyen, H.T.; Le, H.M.; Nguyen, A.T.; Dinh, N.T.T.; Hoang, S.A.; Van $\mathrm{Ha}, \mathrm{C}$. Copper nanoparticle application enhances plant growth and grain yield in maize under drought stress conditions. J. Plant Growth Regul. 2021, 1-12. [CrossRef] 
33. Nawaz, F.; Naeem, M.; Akram, A.; Ashraf, M.Y.; Ahmad, K.S.; Zulfiqar, B.; Sardar, H.; Shabbir, R.N.; Majeed, S.; Shehzad, M.A.; et al. Seed priming with $\mathrm{KNO}_{3}$ mediates biochemical processes to inhibit lead toxicity in maize (Zea mays L.). J. Sci. Food Agric. 2017, 97, 4780-4789. [CrossRef]

34. Imran, M.; Boelt, B.; Mühling, K.-H. Zinc seed priming improves salt resistance in maize. J. Agron. Crop. Sci. 2018, 204, 390-399. [CrossRef]

35. Basit, A.; Hussain, S.; Abid, M.; Zafar-Ul-Hye, M.; Ahmed, N. Zinc and potassium priming of maize (Zea mays L.) seeds for salt-affected soils. J. Plant Nutr. 2021, 44, 130-141. [CrossRef]

36. Khan, I.; Raza, M.A.; Awan, S.A.; Shah, G.A.; Rizwan, M.; Ali, B.; Tariq, R.; Hassan, M.J.; Alyemeni, M.N.; Brestic, M.; et al. Amelioration of salt induced toxicity in pearl millet by seed priming with silver nanoparticles (AgNPs): The oxidative damage, antioxidant enzymes and ions uptake are major determinants of salt tolerant capacity. Plant Physiol. Biochem. 2020, 156, 221-232. [CrossRef] [PubMed]

37. Rojek, J.; Kozieradzka-Kiszkurno, M.; Kapusta, M.; Aksmann, A.; Jacewicz, D.; Drzezdzon, J.; Tesmar, A.; Zamojc, K.; Wyrzykowski, D.; Chmurzynski, L. The effect of vanadium(IV) complexes on development of Arabidopsis thaliana subjected to $\mathrm{H}_{2} \mathrm{O}_{2}$-induced stress. Funct. Plant Biol. 2019, 46, 942. [CrossRef] [PubMed]

38. Singh, A.; Banerjee, A.; Roychoudhury, A. Seed priming with calcium compounds abrogate fluoride-induced oxidative stress by upregulating defence pathways in an indica rice variety. Protoplasma 2019, 257, 767-782. [CrossRef] [PubMed]

39. Winter, T.R.; Borkowski, L.; Zeier, J.; Rostás, M. Heavy metal stress can prime for herbivore-induced plant volatile emission. Plant Cell Environ. 2012, 35, 1287-1298. [CrossRef] [PubMed]

40. Morkunas, I.; Woźniak, A.; Mai, V.; Rucińska-Sobkowiak, R.; Jeandet, P. The role of heavy metals in plant response to biotic stress. Molecules 2018, 23, 2320. [CrossRef]

41. Carreiras, J.; Perez-Romero, J.A.; Mateos-Naranjo, E.; Redondo, S.; Matos, A.R.; Cacador, I.; Duarte, B. The effect of heavy metal contamination pre-conditioning in the heat stress tolerance of native and invasive Mediterranean halophytes. Ecol. Indic. 2020, 111, 106045. [CrossRef]

42. Chen, K.; Arora, R. Priming memory invokes seed stress-tolerance. Environ. Exp. Bot. 2013, 94, 33-45. [CrossRef]

43. Paparella, S.; Araújo, S.S.; Rossi, G.; Wijayasinghe, M.; Carbonera, D.; Balestrazzi, A. Seed priming: State of the art and new perspectives. Plant Cell Rep. 2015, 34, 1281-1293. [CrossRef]

44. Forti, C.; Shankar, A.; Singh, A.; Balestrazzi, A.; Prasad, V.; Macovei, A. Hydropriming and biopriming improve Medicago truncatula seed germination and upregulate DNA repair and antioxidant genes. Genes 2020, 11, 242. [CrossRef]

45. Babajani, A.; Iranbakhsh, A.; Ardebili, Z.O.; Eslami, B. Seed priming with non-thermal plasma modified plant reactions to selenium or zinc oxide nanoparticles: Cold plasma as a novel emerging tool for plant science. Plasma Chem. Plasma Process. 2018, 39, 21-34. [CrossRef]

46. Waqas, M.; Korres, N.E.; Khan, M.D.; Nizami, A.-S.; Deeba, F.; Ali, I.; Hussain, H. Advances in the concept and methods of seed priming. In Priming and Pretreatment of Seeds and Seedlings; Springer: Singapore, 2019; pp. 11-41.

47. Bhardwaj, J.; Anand, A.; Nagarajan, S. Biochemical and biophysical changes associated with magnetopriming in germinating cucumber seeds. Plant Physiol. Biochem. 2012, 57, 67-73. [CrossRef]

48. Noutoshi, Y.; Okazaki, M.; Kida, T.; Nishina, Y.; Morishita, Y.; Ogawa, T.; Suzuki, H.; Shibata, D.; Jikumaru, Y.; Hanada, A.; et al. Novel plant immune-priming compounds identified via high-throughput chemical screening target salicylic acid glucosyltransferases in Arabidopsis. Plant Cell 2012, 24, 3795-3804. [CrossRef] [PubMed]

49. Demecsová, L.; Zelinová, V.; Liptáková, L'.; Valentovičová, K.; Tamás, L. Indole-3-butyric acid priming reduced cadmium toxicity in barley root tip via NO generation and enhanced glutathione peroxidase activity. Planta 2020, 252, 1-16. [CrossRef] [PubMed]

50. Sytar, O.; Kumari, P.; Yadav, S.; Brestic, M.; Rastogi, A. Phytohormone priming: Regulator for heavy metal stress in plants. J. Plant Growth Regul. 2019, 38, 739-752. [CrossRef]

51. Kobylińska, A.; Reiter, R.J.; Posmyk, M.M. Melatonin protects cultured tobacco cells against lead-induced cell death via inhibition of cytochrome c translocation. Front. Plant Sci. 2017, 8, 1560. [CrossRef]

52. Viviane, C.; Sharella, S.; Kees, H.; Hervé, D.d.B.; Filip, C.; Inge, H.; Jos, M.R.; Víctor, J.C. Priming of plant growth promotion by volatiles of root-associated Microbacterium spp. Appl. Environ. Microbiol. 2018, 84, 1865-1883.

53. Moghanloo, M.; Iranbakhsh, A.; Ebadi, M.; Satari, T.N.; Ardebili, Z.O. Seed priming with cold plasma and supplementation of culture medium with silicon nanoparticle modified growth, physiology, and anatomy in Astragalus fridae as an endangered species. Acta Physiol. Plant. 2019, 41, 54. [CrossRef]

54. Nowak, J.; Shulaev, V. Priming for transplant stress resistance in in vitro propagation. Vitr. Cell. Dev. Biol. Anim. 2003, 39, 107-124. [CrossRef]

55. Da Silva, J.A.T.; Singh, N.; Tanaka, M. Priming biotic factors for optimal protocorm-like body and callus induction in hybrid Cymbidium (Orchidaceae), and assessment of cytogenetic stability in regenerated plantlets. Plant Cell Tissue Organ Cult. (PCTOC) 2006, 84, 135-144. [CrossRef]

56. Ray, A.; Bhattacharya, S. An improved micropropagation of Eclipta alba by in vitro priming with chlorocholine chloride. Plant Cell, Tissue Organ Cult. 2007, 92, 315-319. [CrossRef]

57. Rezaei, F.; Kartal, M.; Erdem, S.A. Effect of priming on thymoquinone content and in vitro plant regeneration with tissue culture of black cumin (Nigella sativa L.) seeds. J. Chem. Metrol. 2018, 12, 98. [CrossRef] 
58. Paulert, R.; Ebbinghaus, D.; Urlass, C.; Moerschbacher, B.M. Priming of the oxidative burst in rice and wheat cell cultures by ulvan, a polysaccharide from green macroalgae, and enhanced resistance against powdery mildew in wheat and barley plants. Plant Pathol. 2010, 59, 634-642. [CrossRef]

59. Mhlongo, M.I.; Piater, L.A.; Steenkamp, P.A.; Madala, N.E.; Dubery, I.A. Priming agents of plant defense stimulate the accumulation of mono- and di-acylated quinic acids in cultured tobacco cells. Physiol. Mol. Plant Pathol. 2014, 88, 61-66. [CrossRef]

60. Gangopadhyay, M.; Gantait, S.; Palchoudhury, S.; Ali, N.; Mondal, C.; Pal, A.K. UVC-priming mediated modulation of forskolin biosynthesis key genes against Macrophomina root rot of Coleus forskohlii - a tissue culture based sustainable approach. Phytochem. Lett. 2016, 17, 36-44. [CrossRef]

61. Ding, M.; Wang, X.; Li, Y. Acquired tolerance to cadmium following long-term acclimation to $\mathrm{CdCl}_{2}$ in rice suspension cultures. Plant Cell Tissue Organ Cult. 2016, 124, 47-55. [CrossRef]

62. Chun, H.J.; Baek, D.; Cho, H.M.; Jung, H.S.; Jeong, M.S.; Jung, W.-H.; Choi, C.W.; Lee, S.H.; Jin, B.J.; Park, M.S.; et al. Metabolic adjustment of Arabidopsis root suspension cells during adaptation to salt stress and mitotic stress memory. Plant Cell Physiol. 2018, 60, 612-625. [CrossRef]

63. Khairy, A.I.H.; Oh, M.J.; Lee, S.M.; Kim, D.S.; Roh, K.S. Nitric oxide overcomes Cd and Cu toxicity in in vitro-grown tobacco plants through increasing contents and activities of rubisco and rubisco activase. Biochim. Open 2016, 2, 41-51. [CrossRef]

64. Petrov, V.D.; Van Breusegem, F. Hydrogen peroxide-a central hub for information flow in plant cells. AoB Plants 2012, 2012, pls014. [CrossRef] [PubMed]

65. He, H.; Li, Y.; He, L.-F. The central role of hydrogen sulfide in plant responses to toxic metal stress. Ecotoxicol. Environ. Saf. 2018, 157, 403-408. [CrossRef] [PubMed]

66. Corpas, F.J.; González-Gordo, S.; Cañas, A.; Palma, J.M. Nitric oxide and hydrogen sulfide in plants: Which comes first? J. Exp. Bot. 2019, 70, 4391-4404. [CrossRef] [PubMed]

67. Luo, S.; Calderón-Urrea, A.; Yu, J.; Liao, W.; Xie, J.; Lv, J.; Feng, Z.; Tang, Z. The role of hydrogen sulfide in plant alleviates heavy metal stress. Plant Soil 2020, 449, 1-10. [CrossRef]

68. Wang, H.; Ji, F.; Zhang, Y.; Hou, J.; Liu, W.; Huang, J.; Liang, W. Interactions between hydrogen sulphide and nitric oxide regulate two soybean citrate transporters during the alleviation of aluminum toxicity. Plant Cell Environ. 2019, 42, 2340-2356. [CrossRef] [PubMed]

69. Zhang, J.; Zhou, M.; Zhou, H.; Zhao, D.; Gotor, C.; Romero, L.C.; Shen, J.; Ge, Z.; Zhang, Z.; Shen, W.; et al. Hydrogen sulfide $\left(\mathrm{H}_{2} \mathrm{~S}\right)$, a signaling molecule in plant stress responses. J. Integr. Plant Biol 2020, 63, 146-160. [CrossRef]

70. Hossain, M.A.; Bhattacharjee, S.; Armin, S.-M.; Qian, P.; Xin, W.; Li, H.-Y.; Burritt, D.J.; Fujita, M.; Tran, L.P. Hydrogen peroxide priming modulates abiotic oxidative stress tolerance: Insights from ROS detoxification and scavenging. Front. Plant Sci. 2015, 6, 420. [CrossRef]

71. Cuypers, A.; Hendrix, S.; Amaral dos Reis, R.; De Smet, S.; Deckers, J.; Gielen, H.; Jozefczak, M.; Loix, C.; Vercampt, H.; Vangronsveld, J.; et al. Hydrogen peroxide, signaling in disguise during metal phytotoxicity. Front. Plant Sci. 2016, 7, 470. [CrossRef]

72. Khan, M.I.R.; Khan, N.A.; Masood, A.; Per, T.S.; Asgher, M. Hydrogen peroxide alleviates nickel-inhibited photosynthetic responses through increase in use-efficiency of nitrogen and sulfur, and glutathione production in mustard. Front. Plant Sci. 2016, 7, 44. [CrossRef]

73. Nazir, F.; Hussain, A.; Fariduddin, Q. Hydrogen peroxide modulate photosynthesis and antioxidant systems in tomato (Solanum lycopersicum L.) plants under copper stress. Chemosphere 2019, 230, 544-558. [CrossRef]

74. Xu, F.J.; Jin, C.W.; Liu, W.J.; Zhang, Y.S.; Lin, X.Y. Pretreatment with $\mathrm{H}_{2} \mathrm{O}_{2}$ alleviates aluminum-induced oxidative stress in wheat seedlings. J. Integr. Plant Biol. 2010, 53, 44-53. [CrossRef]

75. Asgher, M.; Ahmed, S.; Sehar, Z.; Gautam, H.; Gandhi, S.G.; Khan, N.A. Hydrogen peroxide modulates activity and expression of antioxidant enzymes and protects photosynthetic activity from arsenic damage in rice (Oryza sativa L.). J. Hazard. Mater. 2021, 401, 123365. [CrossRef]

76. Bai, X.-J.; Liu, L.-J.; Zhang, C.-H.; Ge, Y.; Cheng, W.-D. Effect of $\mathrm{H}_{2} \mathrm{O}_{2}$ Pretreatment on Cd tolerance of different rice cultivars. Rice Sci. 2011, 18, 29-35. [CrossRef]

77. Yıldız, M.; Terzi, H.; Bingül, N.; Yıldız, M. Protective role of hydrogen peroxide pretreatment on defense systems and BnMP1 gene expression in $\mathrm{Cr}(\mathrm{VI})$-stressed canola seedlings. Ecotoxicology 2013, 22, 1303-1312. [CrossRef]

78. Kabała, K.; Zboińska, M.; Głowiak, D.; Reda, M.; Jakubowska, D.; Janicka, M. Interaction between the signaling molecules hydrogen sulfide and hydrogen peroxide and their role in vacuolar $\mathrm{H}^{+}$-ATPase regulation in cadmium-stressed cucumber roots. Physiol. Plant. 2019, 166, 688-704. [CrossRef]

79. Hasanuzzaman, M.; Nahar, K.; Gill, S.S.; Alharby, H.F.; Razafindrabe, B.H.N.; Fujita, M. Hydrogen peroxide pretreatment mitigates cadmium-induced oxidative stress in Brassica napus L.: An intrinsic study on antioxidant defense and glyoxalase systems. Front. Plant Sci. 2017, 8, 115. [CrossRef] [PubMed]

80. Wen, J.-F.; Gong, M.; Liu, Y.; Hu, J.-L.; Deng, M.-H. Effect of hydrogen peroxide on growth and activity of some enzymes involved in proline metabolism of sweet corn seedlings under copper stress. Sci. Hortic. 2013, 164, 366-371. [CrossRef]

81. Kopyra, M.; Gwóźdź, E.A. Nitric oxide stimulates seed germination and counteracts the inhibitory effect of heavy metals and salinity on root growth of Lupinus luteus. Plant Physiol. Biochem. 2003, 41, 1011-1017. [CrossRef] 
82. Yang, L.; Ji, J.; Harris-Shultz, K.R.; Wang, H.; Wang, H.; Abd-Allah, E.F.; Luo, Y.; Hu, X. The dynamic changes of the plasma membrane proteins and the protective roles of nitric oxide in rice subjected to heavy metal cadmium stress. Front. Plant Sci. 2016, 7, 190. [CrossRef] [PubMed]

83. Liu, S.; Yang, R.; Pan, Y.; Ma, M.; Pan, J.; Zhao, Y.; Cheng, Q.; Wu, M.; Wang, M.; Zhang, L. Nitric oxide contributes to minerals absorption, proton pumps and hormone equilibrium under cadmium excess in Trifolium repens L. plants. Ecotoxicol. Environ. Saf. 2015, 119, 35-46. [CrossRef]

84. Seabra, A.B.; Oliveira, H.C. How nitric oxide donors can protect plants in a changing environment: What we know so far and perspectives. AIMS Mol. Sci. 2016, 3, 692-718. [CrossRef]

85. Fang, P.; Sun, T.; Wang, Y.; Ding, Y.; Pandey, A.K.; Zhu, C.; Xu, P. Plant gasotransmitters: Light molecules interplaying with heavy metals. In Reviews in Environmental Science and Bio-Technology; Springer Science and Business Media B.V.: New York, NY, USA, 2021; pp. 1-23.

86. Gill, S.S.; Hasanuzzaman, M.; Nahar, K.; Macovei, A.; Tuteja, N. Importance of nitric oxide in cadmium stress tolerance in crop plants. Plant Physiol. Biochem. 2013, 63, 254-261. [CrossRef]

87. Kaur, G.; Singh, H.P.; Batish, D.R.; Mahajan, P.; Kohli, R.K.; Rishi, V. Exogenous nitric oxide (NO) interferes with lead (Pb)-induced toxicity by detoxifying reactive oxygen species in hydroponically grown wheat (Triticum aestivum) roots. PLoS ONE 2015, 10, e0138713. [CrossRef] [PubMed]

88. Rizwan, M.; Mostofa, M.G.; Ahmad, M.Z.; Imtiaz, M.; Mehmood, S.; Adeel, M.; Dai, Z.; Li, Z.; Aziz, O.; Zhang, Y.; et al. Nitric oxide induces rice tolerance to excessive nickel by regulating nickel uptake, reactive oxygen species detoxification and defense-related gene expression. Chemosphere 2018, 191, 23-35. [CrossRef] [PubMed]

89. Kaya, C.; Ashraf, M.; Alyemeni, M.N.; Ahmad, P. Responses of nitric oxide and hydrogen sulfide in regulating oxidative defence system in wheat plants grown under cadmium stress. Physiol. Plant. 2019, 168, 345-360. [CrossRef] [PubMed]

90. Hasanuzzaman, M.; Fujita, M. Exogenous sodium nitroprusside alleviates arsenic-induced oxidative stress in wheat (Triticum aestivum L.) seedlings by enhancing antioxidant defense and glyoxalase system. Ecotoxicology 2013, 22, 584-596. [CrossRef]

91. Kováčik, J.; Babula, P.; Hedbavny, J.; Švec, P. Manganese-induced oxidative stress in two ontogenetic stages of chamomile and amelioration by nitric oxide. Plant Sci. 2014, 215-216, 1-10. [CrossRef]

92. Dong, Y.; Chen, W.; Xu, L.; Kong, J.; Liu, S.; He, Z. Nitric oxide can induce tolerance to oxidative stress of peanut seedlings under cadmium toxicity. Plant Growth Regul. 2016, 79, 19-28. [CrossRef]

93. Rey, P.; Tarrago, L. Physiological roles of plant methionine sulfoxide reductases in redox homeostasis and signaling. Antioxidants 2018, 7, 114. [CrossRef]

94. Xiong, J.; An, L.; Lu, H.; Zhu, C. Exogenous nitric oxide enhances cadmium tolerance of rice by increasing pectin and hemicellulose contents in root cell wall. Planta 2009, 230, 755-765. [CrossRef]

95. Lisjak, M.; Teklic, T.; Wilson, I.D.; Whiteman, M.; Hancock, J.T. Hydrogen sulfide: Environmental factor or signalling molecule? Plant Cell Environ. 2013, 36, 1607-1616. [CrossRef]

96. Shan, C.; Dai, H.; Sun, Y. Hydrogen sulfide protects wheat seedlings against copper stress by regulating the ascorbate and glutathione metabolism in leaves. Aust. J. Crop Sci. 2012, 6, 248-254.

97. Valivand, M.; Amooaghaie, R.; Ahadi, A. Seed priming with $\mathrm{H}_{2} \mathrm{~S}$ and $\mathrm{Ca}^{2+}$ trigger signal memory that induces cross-adaptation against nickel stress in zucchini seedlings. Plant Physiol. Biochem. 2019, 143, 286-298. [CrossRef]

98. Zanganeh, R.; Jamei, R.; Rahmani, F. Role of salicylic acid and hydrogen sulfide in promoting lead stress tolerance and regulating free amino acid composition in Zea mays L. Acta Physiol. Plant. 2019, 41, 94. [CrossRef]

99. Ali, S.; Farooq, M.A.; Hussain, S.; Yasmeen, T.; Abbasi, G.; Zhang, G. Alleviation of chromium toxicity by hydrogen sulfide in barley. Environ. Toxicol. Chem. 2013, 32, 2234-2239. [CrossRef]

100. Fu, M.-M.; Dawood, M.; Wang, N.-H.; Wu, F. Exogenous hydrogen sulfide reduces cadmium uptake and alleviates cadmium toxicity in barley. Plant Growth Regul. 2019, 89, 227-237. [CrossRef]

101. Kharbech, O.; Ben Massoud, M.; Sakouhi, L.; Djebali, W.; Mur, L.A.J.; Chaoui, A. Exogenous application of hydrogen sulfide reduces chromium toxicity in maize seedlings by suppressing NADPH oxidase activities and methylglyoxal accumulation. Plant Physiol. Biochem. 2020, 154, 646-656. [CrossRef]

102. Powell, C.R.; Dillon, K.M.; Matson, J.B. A review of hydrogen sulfide $\left(\mathrm{H}_{2} \mathrm{~S}\right)$ donors: Chemistry and potential therapeutic applications. Biochem. Pharmacol. 2018, 149, 110-123. [CrossRef] [PubMed]

103. Carter, J.M.; Brown, E.M.; Grace, J.P.; Salem, A.K.; Irish, E.E.; Bowden, N.B. Improved growth of pea, lettuce, and radish plants using the slow release of hydrogen sulfide from GYY-4137. PLoS ONE 2018, 13, e0208732. [CrossRef]

104. Calderone, V.; Martelli, A.; Testai, L.; Citi, V.; Breschi, M.C. Using hydrogen sulfide to design and develop drugs. Expert Opin. Drug Discov. 2015, 11, 163-175. [CrossRef]

105. Popova, L.P.; Maslenkova, L.T.; Ivanova, A.; Stoinova, Z.G. Role of salicylic acid in alleviating heavy metal stress. In Environmental Adaptations and Stress Tolerance of Plants in the Era of Climate Change; Springer: New York, NY, USA, 2011; pp. $447-466$.

106. Gharbi, E.; Martínez, J.P.; Benahmed, H.; Dailly, H.; Quinet, M.; Lutts, S. The salicylic acid analog 2,6-dichloroisonicotinic acid has specific impact on the response of the halophyte plant species Solanum chilense to salinity. Plant Growth Regul. 2017, 82, 517-525. [CrossRef] 
107. Khan, F.; Hussain, S.; Tanveer, M.; Khan, S.; Hussain, H.A.; Iqbal, B.; Geng, M. Coordinated effects of lead toxicity and nutrient deprivation on growth, oxidative status, and elemental composition of primed and non-primed rice seedlings. Environ. Sci. Pollut. Res. 2018, 25, 21185-21194. [CrossRef]

108. Khan, F.; Hussain, S.; Khan, S.; Geng, M. Seed priming improved antioxidant defense system and alleviated Ni-induced adversities in rice seedlings under N, P, or K deprivation. Front. Plant Sci. 2020, 11, 1. [CrossRef] [PubMed]

109. Maghsoudi, K.; Arvin, M.J.; Ashraf, M. Mitigation of arsenic toxicity in wheat by the exogenously applied salicylic acid, 24-epi-brassinolide and silicon. J. Soil Sci. Plant Nutr. 2019, 20, 577-588. [CrossRef]

110. Safari, F.; Akramian, M.; Salehi-Arjmand, H.; Khadivi, A. Physiological and molecular mechanisms underlying salicylic acidmitigated mercury toxicity in lemon balm (Melissa officinalis L.). Ecotoxicol. Environ. Saf. 2019, 183, 109542. [CrossRef] [PubMed]

111. Agami, R.A.; Mohamed, G.F. Exogenous treatment with indole-3-acetic acid and salicylic acid alleviates cadmium toxicity in wheat seedlings. Ecotoxicol. Environ. Saf. 2013, 94, 164-171. [CrossRef]

112. Karalija, E.; Selović, A.; Dahija, S.; Demir, A.; Samardžić, J.; Vrobel, O.; Parić, A. Use of seed priming to improve Cd accumulation and tolerance in Silene sendtneri, novel Cd hyper-accumulator. Ecotoxicol. Environ. Saf. 2021, 210, 111882. [CrossRef]

113. Belkadhi, A.; De Haro, A.; Obregon, S.; Chaibi, W.; Djebali, W. Positive effects of salicylic acid pretreatment on the composition of flax plastidal membrane lipids under cadmium stress. Environ. Sci. Pollut. Res. 2014, 22, 1457-1467. [CrossRef] [PubMed]

114. Belkhadi, A.; Hediji, H.; Abbes, Z.; Nouairi, I.; Barhoumi, Z.; Zarrouk, M.; Chaibi, W.; Djebali, W. Effect of exogenous salicylic acid pre-treatment on cadmium toxicity and leaf lipid content in Linum usitatissimum L. Ecotoxicol Environ. Saf. 2010, 73, 1004-1011. [CrossRef]

115. Tao, S.; Sun, L.; Ma, C.; Li, L.; Li, G.; Hao, L. Reducing basal salicylic acid enhances Arabidopsis tolerance to lead or cadmium. Plant Soil 2013, 372, 309-318. [CrossRef]

116. Wiszniewska, A.; Koźmińska, A.; Hanus-Fajerska, E.; Dziurka, M.; Dziurka, K. Insight into mechanisms of multiple stresses tolerance in a halophyte Aster tripolium subjected to salinity and heavy metal stress. Ecotoxicol. Environ. Saf. 2019, 180, 12-22. [CrossRef]

117. Takahashi, T.; Kakehi, J.-I. Polyamines: Ubiquitous polycations with unique roles in growth and stress responses. Ann. Bot. 2009, 105, 1-6. [CrossRef]

118. Bae, D.-H.; Lane, D.J.; Jansson, P.J.; Richardson, D.R. The old and new biochemistry of polyamines. Biochim. Biophys. Acta Gen. Subj. 2018, 1862, 2053-2068. [CrossRef]

119. Paul, S.; Banerjee, A.; Roychoudhury, A. Role of polyamines in mediating antioxidant defense and epigenetic regulation in plants exposed to heavy metal toxicity. In Plants under Metal and Metalloid Stress; Springer: Singapore, 2018; pp. $229-247$.

120. Hasanuzzaman, M.; Alhaithloul, H.A.S.; Parvin, K.; Bhuyan, M.H.M.B.; Tanveer, M.; Mohsin, S.M.; Nahar, K.; Soliman, M.H.; Mahmud, J.A.; Fujita, M. Polyamine action under metal/metalloid stress: Regulation of biosynthesis, metabolism, and molecular Interactions. Int. J. Mol. Sci. 2019, 20, 3215. [CrossRef] [PubMed]

121. Takahashi, T.; Tong, W. Regulation and diversity of polyamine biosynthesis in plants. In Polyamines: A Universal Molecular Nexus for Growth, Survival, and Specialized Metabolism; Springer: Tokyo, Japan, 2014; pp. 27-44.

122. Taie, H.A.A.; El-Yazal, M.A.S.; Ahmed, S.M.A.; Rady, M.M. Polyamines modulate growth, antioxidant activity, and genomic DNA in heavy metal-stressed wheat plant. Environ. Sci. Pollut. Res. 2019, 26, 22338-22350. [CrossRef] [PubMed]

123. Choudhary, S.P.; Kanwar, M.; Bhardwaj, R.; Yu, J.-Q.; Tran, L.-S.P. Chromium stress mitigation by polyamine-brassinosteroid application involves phytohormonal and physiological strategies in Raphanus sativus L. PLoS ONE 2012, 7, e33210. [CrossRef] [PubMed]

124. Agami, R.A. Pre-soaking in indole-3-acetic acid or spermidine enhances copper tolerance in wheat seedlings. South Afr. J. Bot. 2016, 104, 167-174. [CrossRef]

125. Altaf, M.M.; Shahid, R.; Ren, M.; Mora-Poblete, F.; Arnao, M.B.; Naz, S.; Anwar, M.; Shahid, S.; Shakoor, A.; Sohail, H.; et al. Phytomelatonin: An overview of the importance and mediating functions of melatonin against environmental stresses. Physiol. Plant. 2020, 1-27. [CrossRef]

126. Sun, C.; Liu, L.; Wang, L.; Li, B.; Jin, C.; Lin, X. Melatonin: A master regulator of plant development and stress responses. J. Integr. Plant Biol. 2021, 63, 126-145. [CrossRef] [PubMed]

127. Yang, X.L.; Xu, H.; Li, D.; Gao, X.; Li, T.L.; Wang, R. Effect of melatonin priming on photosynthetic capacity of tomato leaves under low-temperature stress. Photosynthetica 2017, 56, 884-892. [CrossRef]

128. Khan, M.N.; Zhang, J.; Luo, T.; Liu, J.; Rizwan, M.; Fahad, S.; Xu, Z.; Hu, L. Seed priming with melatonin coping drought stress in rapeseed by regulating reactive oxygen species detoxification: Antioxidant defense system, osmotic adjustment, stomatal traits and chloroplast ultrastructure perseveration. Ind. Crop. Prod. 2019, 140, 111597. [CrossRef]

129. Nawaz, M.A.; Jiao, Y.; Chen, C.; Shireen, F.; Zheng, Z.; Imtiaz, M.; Bie, Z.; Huang, Y. Melatonin pretreatment improves vanadium stress tolerance of watermelon seedlings by reducing vanadium concentration in the leaves and regulating melatonin biosynthesis and antioxidant-related gene expression. J. Plant Physiol. 2018, 220, 115-127. [CrossRef]

130. Xie, C.; Xiong, X.; Huang, Z.; Sun, L.; Ma, J.; Cai, S.; Yu, F.; Zhong, W.; Chen, S.; Li, X. Exogenous melatonin improves lead tolerance of bermudagrass through modulation of the antioxidant defense system. Int. J. Phytoremed. 2018, 20, 1408-1417. [CrossRef]

131. Shah, A.A.; Ahmed, S.; Ali, A.; Yasin, N.A. 2-Hydroxymelatonin mitigates cadmium stress in Cucumis sativus seedlings: Modulation of antioxidant enzymes and polyamines. Chemosphere 2020, 243, 125308. [CrossRef] 
132. Ni, J.; Wang, Q.; Shah, F.A.; Liu, W.; Wang, D.; Huang, S.; Fu, S.; Wu, L. Exogenous melatonin confers cadmium tolerance by counterbalancing the hydrogen peroxide homeostasis in wheat seedlings. Molecules 2018, 23, 799. [CrossRef] [PubMed]

133. Tousi, S.; Zoufan, P.; Ghahfarrokhie, A.R. Alleviation of cadmium-induced phytotoxicity and growth improvement by exogenous melatonin pretreatment in mallow (Malva parviflora) plants. Ecotoxicol. Environ. Saf. 2020, 206, 111403. [CrossRef]

134. Abd_Allah, E.F.; Hashem, A.; Alam, P.; Ahmad, P. Silicon alleviates nickel-induced oxidative stress by regulating antioxidant defense and glyoxalase systems in mustard plants. J. Plant Growth Regul. 2019, 38, 1260-1273. [CrossRef]

135. Kaya, C.; Akram, N.A.; Ashraf, M.; Alyemeni, M.N.; Ahmad, P. Exogenously supplied silicon (Si) improves cadmium tolerance in pepper (Capsicum annuum L.) by up-regulating the synthesis of nitric oxide and hydrogen sulfide. J. Biotechnol. 2020, 316, 35-45. [CrossRef] [PubMed]

136. Moulick, D.; Santra, S.C.; Ghosh, D. Effect of selenium induced seed priming on arsenic accumulation in rice plant and subsequent transmission in human food chain. Ecotoxicol. Environ. Saf. 2018, 152, 67-77. [CrossRef] [PubMed]

137. Ragab, G.; Saad-Allah, K. Seed priming with greenly synthesized sulfur nanoparticles enhances antioxidative defense machinery and restricts oxidative injury under manganese stress in Helianthus annuus (L.) seedlings. J. Plant Growth Regul. 2020, 1-9. [CrossRef]

138. Fatemi, H.; Pour, B.E.; Rizwan, M. Foliar application of silicon nanoparticles affected the growth, vitamin C, flavonoid, and antioxidant enzyme activities of coriander (Coriandrum sativum L.) plants grown in lead (Pb)-spiked soil. Environ. Sci. Pollut. Res. 2021, 28, 1417-1425. [CrossRef]

139. Sotoodehnia-Korani, S.; Iranbakhsh, A.; Ebadi, M.; Majd, A.; Ardebili, Z.O. Selenium nanoparticles induced variations in growth, morphology, anatomy, biochemistry, gene expression, and epigenetic DNA methylation in Capsicum annuum; an in vitro study. Environ. Pollut. 2020, 265, 114727. [CrossRef]

140. Frost, C.J.; Mescher, M.C.; Dervinis, C.; Davis, J.M.; Carlson, J.E.; de Moraes, C.M. Priming defense genes and metabolites in hybrid poplar by the green leaf volatile cis -3-hexenyl acetate. New Phytol. 2008, 180, 722-734. [CrossRef]

141. Conrath, U. Molecular aspects of defence priming. Trends Plant Sci. 2011, 16, 524-531. [CrossRef]

142. Kubala, S.; Garnczarska, M.; Wojtyla, L.; Clippe, A.; Kosmala, A.; Zmienko, A.; Lutts, S.; Quinet, M. Deciphering priming-induced improvement of rapeseed (Brassica napus L.) germination through an integrated transcriptomic and proteomic approach. Plant Sci. 2015, 231, 94-113. [CrossRef]

143. Mo, H.-J.; Sun, Y.-X.; Zhu, X.-L.; Wang, X.-F.; Zhang, Y.; Yang, J.; Yan, G.-J.; Ma, Z.-Y. Cotton S-adenosylmethionine decarboxylasemediated spermine biosynthesis is required for salicylic acid- and leucine-correlated signaling in the defense response to Verticillium dahliae. Planta 2016, 243, 1023-1039. [CrossRef]

144. Qi, C.; Zhang, H.; Liu, Y.; Wang, X.; Dong, D.; Yuan, X.; Li, X.; Zhang, X.; Li, X.; Zhang, N.; et al. CsSNAT positively regulates salt tolerance and growth of cucumber by promoting melatonin biosynthesis. Environ. Exp. Bot. 2020, 175, 104036. [CrossRef]

145. Hu, T.; Liu, S.Q.; Amombo, E.; Fu, J.M. Stress memory induced rearrangements of HSP transcription, photosystem II photochemistry and metabolism of tall fescue (Festuca arundinacea Schreb.) in response to high-temperature stress. Front. Plant Sci. 2015, 6, 403. [CrossRef]

146. De Camargos, L.F.; Fraga, O.T.; Oliveira, C.C.; Da Silva, J.C.F.; Fontes, E.P.B.; Reis, P.A.B. Development and cell death domaincontaining asparagine-rich protein (DCD/NRP): An essential protein in plant development and stress responses. Theor. Exp. Plant Physiol. 2019, 31, 59-70. [CrossRef]

147. Ederli, L.; Reale, L.; Madeo, L.; Ferranti, F.; Gehring, C.; Fornaciari, M.; Romano, B.; Pasqualini, S. NO release by nitric oxide donors in vitro and in planta. Plant Physiol. Biochem. 2009, 47, 42-48. [CrossRef]

148. Lefevere, H.; Bauters, L.; Gheysen, G. Salicylic acid biosynthesis in plants. Front. Plant Sci. 2020, 11, 338. [CrossRef]

149. Liu, J.-H.; Nada, K.; Honda, C.; Kitashiba, H.; Wen, X.-P.; Pang, X.-M.; Moriguchi, T. Polyamine biosynthesis of apple callus under salt stress: Importance of the arginine decarboxylase pathway in stress response. J. Exp. Bot. 2006, 57, 2589-2599. [CrossRef]

150. Lee, K.-W.; Cha, J.-Y.; Kim, K.-H.; Kim, Y.-G.; Lee, B.-H.; Lee, S.-H. Overexpression of alfalfa mitochondrial HSP23 in prokaryotic and eukaryotic model systems confers enhanced tolerance to salinity and arsenic stress. Biotechnol. Lett. 2011, 34, 167-174. [CrossRef] [PubMed]

151. Santhanagopalan, I.; Basha, E.; Ballard, K.N.; Bopp, N.E.; Vierling, E. Model Chaperones: Small heat shock proteins from plants. In Heat Shock Proteins; Springer International Publishing: Berlin/Heidelberg, Germany, 2015; Volume 8, pp. $119-153$.

152. Lee, S.-H.; Lee, K.-W.; Lee, D.-G.; Son, D.; Park, S.J.; Kim, K.-Y.; Park, H.S.; Cha, J.-Y. Identification and functional characterization of Siberian wild rye (Elymus sibiricus L.) small heat shock protein 16.9 gene (EsHsp16.9) conferring diverse stress tolerance in prokaryotic cells. Biotechnol. Lett. 2015, 37, 881-890. [CrossRef] [PubMed]

153. Lee, K.-W.; Choi, G.J.; Kim, K.-Y.; Ji, H.J.; Park, H.S.; Kim, Y.-G.; Lee, B.H.; Lee, S.-H. Transgenic expression of MsHsp23 confers enhanced tolerance to abiotic stresses in tall fescue. Asian Australas. J. Anim. Sci. 2012, 25, 818-823. [CrossRef] [PubMed]

154. Lee, J.; Ahn, Y.-J. Heterologous expression of a carrot small heat shock protein increased Escherichia coli viability under lead and arsenic stresses. HortScience 2013, 48, 1323-1326. [CrossRef]

155. Zhao, L.; Peng, B.; Hernandez-Viezcas, J.A.; Rico, C.; Sun, Y.; Peralta-Videa, J.R.; Tang, X.; Niu, G.; Jin, L.; Varela-Ramirez, A.; et al. Stress response and tolerance of Zea mays to $\mathrm{CeO}_{2}$ nanoparticles: Cross talk among $\mathrm{H}_{2} \mathrm{O}_{2}$, heat shock protein, and lipid peroxidation. ACS Nano 2012, 6, 9615-9622. [CrossRef] [PubMed]

156. Balestrazzi, A.; Confalonieri, M.; Macovei, A.; Donà, M.; Carbonera, D. Genotoxic stress and DNA repair in plants: Emerging functions and tools for improving crop productivity. Plant Cell Rep. 2011, 30, 287-295. [CrossRef] 
157. Koźmińska, A.; Wiszniewska, A.; Hanus-Fajerska, E.; Muszyńska, E. Recent strategies of increasing metal tolerance and phytoremediation potential using genetic transformation of plants. Plant Biotechnol. Rep. 2018, 12, 1-14. [CrossRef]

158. Chen, Y.; Liu, Y.; Ding, Y.; Wang, X.; Xu, J. Overexpression of PtPCS enhances cadmium tolerance and cadmium accumulation in tobacco. Plant Cell, Tissue Organ Cult. 2015, 121, 389-396. [CrossRef]

159. Bertini, L.; Proietti, S.; Focaracci, F.; Sabatini, B.; Caruso, C. Epigenetic control of defense genes following MeJA-induced priming in rice (O. sativa). J. Plant Physiol. 2018, 228, 166-177.

160. Souza, L.A.; Monteiro, C.C.; Carvalho, R.F.; Gratão, P.L.; Azevedo, R.A. Dealing with abiotic stresses: An integrative view of how phytohormones control abiotic stress-induced oxidative stress. Theor. Exp. Plant Physiol. 2017, 29, 109-127. [CrossRef]

161. Singh, S.; Prasad, S.M. IAA alleviates Cd toxicity on growth, photosynthesis and oxidative damages in eggplant seedlings. Plant Growth Regul. 2015, 77, 87-98. [CrossRef]

162. Bashri, G.; Prasad, S.M. Exogenous IAA differentially affects growth, oxidative stress and antioxidants system in Cd stressed Trigonella foenum-graecum L. seedlings: Toxicity alleviation by up-regulation of ascorbate-glutathione cycle. Ecotoxicol. Environ. Saf. 2016, 132, 329-338. [CrossRef]

163. Li, S.-W.; Zeng, X.-Y.; Leng, Y.; Feng, L.; Kang, X.-H. Indole-3-butyric acid mediates antioxidative defense systems to promote adventitious rooting in mung bean seedlings under cadmium and drought stresses. Ecotoxicol. Environ. Saf. 2018, 161, 332-341. [CrossRef]

164. Khan, M.Y.; Prakash, V.; Yadav, V.; Chauhan, D.K.; Prasad, S.M.; Ramawat, N.; Singh, V.P.; Tripathi, D.K.; Sharma, S. Regulation of cadmium toxicity in roots of tomato by indole acetic acid with special emphasis on reactive oxygen species production and their scavenging. Plant Physiol. Biochem. 2019, 142, 193-201. [CrossRef]

165. Zhang, C.; He, Q.; Wang, M.; Gao, X.; Chen, J.; Shen, C. Exogenous indole acetic acid alleviates Cd toxicity in tea (Camellia sinensis). Ecotoxicol. Environ. Saf. 2020, 190, 110090. [CrossRef]

166. Ochoa, L.; Medina-Velo, I.A.; Barrios, A.C.; Bonilla-Bird, N.J.; Hernandez-Viezcas, J.A.; Peralta-Videa, J.R.; Gardea-Torresdey, J.L. Modulation of $\mathrm{CuO}$ nanoparticles toxicity to green pea (Pisum sativum Fabaceae) by the phytohormone indole-3-acetic acid. Sci. Total. Environ. 2017, 598, 513-524. [CrossRef]

167. Massoud, M.B.; Sakouhi, L.; Karmous, I.; Zhu, Y.; El Ferjani, E.; Sheehan, D.; Chaoui, A. Protective role of exogenous phytohormones on redox status in pea seedlings under copper stress. J. Plant Physiol. 2018, 221, 51-61. [CrossRef] [PubMed]

168. Wang, P.; Yu, W.; Zhang, J.; Rengel, Z.; Xu, J.; Han, Q.; Chen, L.; Li, K.; Yu, Y.; Chen, Q. Auxin enhances aluminum-induced citrate exudation through upregulation of GmMATE and activation of the plasma membrane $\mathrm{H}^{+}$-ATPase in soybean roots. Ann. Bot. 2016, 118, 933-940. [CrossRef] [PubMed]

169. Nazir, F.; Fariduddin, Q.; Hussain, A.; Alam Khan, T. Brassinosteroid and hydrogen peroxide improve photosynthetic machinery, stomatal movement, root morphology and cell viability and reduce $\mathrm{Cu}$-triggered oxidative burst in tomato. Ecotoxicol. Environ. Saf. 2021, 207, 111081. [CrossRef]

170. Shah, A.A.; Ahmed, S.; Abbas, M.; Yasin, N.A. Seed priming with 3-epibrassinolide alleviates cadmium stress in Cucumis sativus through modulation of antioxidative system and gene expression. Sci. Hortic. 2020, 265, 109203. [CrossRef]

171. Galhaut, L.; De Lespinay, A.; Walker, D.J.; Bernal, M.P.; Correal, E.; Lutts, S. Seed priming of Trifolium repens L. improved germination and early seedling growth on heavy metal-contaminated soil. Water, Air, Soil Pollut. 2014, 225, 1905. [CrossRef]

172. Sneideris, L.C.; Gavassi, M.A.; Campos, M.L.; D'Amico-Damião, V.; Carvalho, R.F. Effects of hormonal priming on seed germination of pigeon pea under cadmium stress. An. Acad. Bras. Cienc. 2015, 87, 1847-1852. [CrossRef]

173. Wu, X.; He, J.; Ding, H.; Zhu, Z.; Chen, J.; Xu, S.; Zha, D. Modulation of zinc-induced oxidative damage in Solanum melongena by 6-benzylaminopurine involves ascorbate-glutathione cycle metabolism. Environ. Exp. Bot. 2015, 116, 1-11. [CrossRef]

174. Agnihotri, A.; Seth, C.S. Does jasmonic acid regulate photosynthesis, clastogenecity, and phytochelatins in Brassica juncea L. in response to Pb-subcellular distribution? Chemosphere 2020, 243, 125361. [CrossRef]

175. Tai, Z.; Yin, X.; Fang, Z.; Shi, G.; Lou, L.; Cai, Q. Exogenous GR24 alleviates cadmium toxicity by reducing cadmium uptake in switchgrass (Panicum virgatum) seedlings. Int. J. Environ. Res. Public Health 2017, 14, 852. [CrossRef]

176. Jisha, K.C.; Puthur, J.T. Halopriming of seeds imparts tolerance to $\mathrm{NaCl}$ and PEG induced stress in Vigna radiata (L.) Wilczek varieties. Physiol. Mol. Biol. Plants 2014, 20, 303-312. [CrossRef]

177. Kumar, M.; Pant, B.; Mondal, S.; Bose, B. Hydro and halo priming: Influenced germination responses in wheat Var-HUW-468 under heavy metal stress. Acta Physiol. Plant. 2016, 38, 1-7. [CrossRef]

178. Román-Ponce, B.; Reza-Vázquez, D.M.; Gutiérrez-Paredes, S.; Haro-Cruz, M.D.J.D.; Maldonado-Hernández, J.; Bahena-Osorio, Y.; Santos, P.E.-D.L.; Wang, E.T.; Vásquez-Murrieta, M.S. Plant growth-promoting traits in rhizobacteria of heavy metal-resistant plants and their effects on Brassica nigra seed germination. Pedosphere 2017, 27, 511-526. [CrossRef]

179. Desoky, E.-S.M.; Merwad, A.-R.M.; Semida, W.M.; Ibrahim, S.A.; El-Saadony, M.T.; Rady, M.M. Heavy metals-resistant bacteria (HM-RB): Potential bioremediators of heavy metals-stressed Spinacia oleracea plant. Ecotoxicol. Environ. Saf. 2020, $198,110685$. [CrossRef]

180. Benidire, L.; Pereira, S.I.A.; Castro, P.M.L.; Boularbah, A. Assessment of plant growth promoting bacterial populations in the rhizosphere of metallophytes from the Kettara mine, Marrakech. Environ. Sci. Pollut. Res. 2016, 23, 21751-21765. [CrossRef]

181. Dabral, S.; Yashaswee; Varma, A.; Choudhary, D.K.; Bahuguna, R.N.; Nath, M. Biopriming with Piriformospora indica ameliorates cadmium stress in rice by lowering oxidative stress and cell death in root cells. Ecotoxicol. Environ. Saf. 2019, 186, 109741. [CrossRef] 
182. Hou, L.; Yu, J.; Zhao, L.; He, X. Dark septate endophytes improve the growth and the tolerance of Medicago sativa and Ammopiptanthus mongolicus under cadmium stress. Front. Microbiol. 2020, 10, 3061. [CrossRef]

183. Qadir, M.; Hussain, A.; Hamayun, M.; Shah, M.; Iqbal, A.; Husna; Murad, W. Phytohormones producing rhizobacterium alleviates chromium toxicity in Helianthus annuus L. by reducing chromate uptake and strengthening antioxidant system. Chemosphere 2020, 258, 127386. [CrossRef]

184. Zhou, C.; Zhu, L.; Ma, Z.; Wang, J. Bacillus amyloliquefaciens SAY09 increases cadmium resistance in plants by activation of auxin-mediated signaling pathways. Genes 2017, 8, 173. [CrossRef]

185. Wang, C.; Liu, Z.; Huang, Y.; Zhang, Y.; Wang, X.; Hu, Z. Cadmium-resistant rhizobacterium Bacillus cereus M4 promotes the growth and reduces cadmium accumulation in rice (Oryza sativa L.). Environ. Toxicol. Pharmacol. 2019, 72, 103265. [CrossRef] [PubMed]

186. Shahid, M.; Javed, M.T.; Tanwir, K.; Akram, M.S.; Tazeen, S.K.; Saleem, M.H.; Masood, S.; Mujtaba, S.; Chaudhary, H.J. Plant growth-promoting Bacillus sp. strain SDA-4 confers Cd tolerance by physio-biochemical improvements, better nutrient acquisition and diminished Cd uptake in Spinacia oleracea L. Physiol. Mol. Biol. Plants 2020, 26, 2417-2433. [CrossRef] [PubMed]

187. Da, K.; Nowak, J.; Flinn, B. Potato cytosine methylation and gene expression changes induced by a beneficial bacterial endophyte, Burkholderia phytofirmans strain PsJN. Plant. Physiol. Biochem. 2012, 50, 24-34. [CrossRef]

188. Versluys, M.; Tarkowski, Ł.P.; Ende, W.V.D. Fructans as DAMPs or MAMPs: Evolutionary prospects, cross-tolerance, and multistress resistance potential. Front. Plant Sci. 2017, 7, 1264. [CrossRef]

189. Fodorpataki, L.; Molnar, K.; Tompa, B.; Plugaru, S.R.C. Priming with vitamin U enhances cold tolerance of lettuce (Lactuca sativa L.). Not. Bot. Horti Agrobot. Cluj-Napoca 2019, 47, 592-598. [CrossRef]

190. Kataria, S.; Baghel, L.; Jain, M.; Guruprasad, K. Magnetopriming regulates antioxidant defense system in soybean against salt stress. Biocatal. Agric. Biotechnol. 2019, 18, 101090. [CrossRef]

191. Sobhy, S.; Allah, K.; Kassem, E.; Hafez, E.; Sewelam, N. Seed priming in natural weed extracts represents a promising practice for alleviating lead stress toxicity. Egypt. J. Exp. Boil. 2019, 15, 453. [CrossRef] 